\title{
The tidally disturbed luminous compact blue galaxy Mkn 1087 and its surroundings ${ }^{\star}$
}

\author{
Á. R. López-Sánchez ${ }^{1}$, C. Esteban ${ }^{1}$, and M. Rodríguez ${ }^{2}$ \\ 1 Instituto de Astrofísica de Canarias, 38200, La Laguna, Tenerife, Spain \\ e-mail: angelrls@ll.iac.es \\ 2 Instituto Nacional de Astrofísica, Óptica y Electrónica, Apdo. Postal 51 y 216, 72000 Puebla, Mexico
}

Received 11 June 2004 / Accepted 27 August 2004

\begin{abstract}
We present new broad-band optical and near-infrared CCD imaging together with deep optical intermediateresolution spectroscopy of Mkn 1087 and its surrounding objects. We analyze the morphology and colors of the stellar populations of the brightest objects, some of them star-formation areas, as well as the kinematics, physical conditions and chemical composition of the ionized gas associated with them. Mkn 1087 does not host an Active Galactic Nucleus, but it could be a Luminous Compact Blue Galaxy. Although it was classified as a suspected Wolf-Rayet galaxy, we do not detect the spectral features of these sort of massive stars. Mkn 1087 shows morphological and kinematical features that can be explained assuming that it is in interaction with two nearby galaxies: the bright KPG 103a and a dwarf $\left(M_{B} \sim-18\right)$ star-forming companion. We argue that this dwarf companion is not a tidal object but an external galaxy because of its low metallicity $[12+\log (\mathrm{O} / \mathrm{H})=$ 8.24] with respect to the one derived for $\operatorname{Mkn} 1087[12+\log (\mathrm{O} / \mathrm{H})=8.57]$ and its kinematics. Some of the non-stellar objects surrounding Mkn 1087 are connected by bridges of matter with the main body, host star-formation events and show similar abundances despite their different angular distances. These facts, together their kinematics, suggest that they are tidal dwarf galaxies formed from material stripped from Mkn 1087. A bright star-forming region at the south of Mkn 1087 (knot \#7) does not show indications of being a tidal galaxy or the product of a merging process as suggested in previous works. We argue that Mkn 1087 and its surroundings should be considered a group of galaxies.
\end{abstract}

Key words. galaxies: starburst - galaxies: interactions - galaxies: abundances - Galaxy: kinematics and dynamics galaxies: clusters: individual: Mkn 1087

\section{Introduction}

One of the most controversial issues about $\mathrm{H}$ II galaxies is the triggering mechanism of the violent bursts of star formation that are observed in them. The estimated star formation rates in these starburst galaxies are so high that the material available for the creation of new stars would be exhausted very soon compared to the age of the universe. This problem is more marked in dwarf galaxies. Thuan (1991) suggested that the bursts have an intermittent behavior. However, star formation episodes show a large variability in duration, ranging from $10^{7}-10^{8}$ year (Rieke \& Lebofsky 1985) to more than $10^{9} \mathrm{yr}$ (Hunter \& Gallagher 1985). In fact, the age of the bursts and the galaxy that hosts them is also a controversial problem. The knowledge of both the triggering mechanism and

$\star$ Based on observations made with several telescopes operated on the islands of La Palma and Tenerife by the Isaac Newton Group of Telescopes and Instituto de Astrofísica de Canarias in the Spanish Observatories of Roque de Los Muchachos and Teide of the Instituto de Astrofísica de Canarias. the age of the starbursts would help to understand the evolution of the galaxies.

Hierarchical formation models of galaxies (Kauffmann et al. 1997) predict that most galaxies have formed by merging of small clouds of protogalactic gas. In this sense, studies of metal deficient blue compact dwarf galaxies (BCDGs) embedded in large presumably primordial H I clouds provide excellent clues about the trigger and youth of the galaxies (Thuan et al. 1995; Izotov \& Thuan 1999) although most of them show an underlying old stellar component (Noeske et al. 2003). Some authors (Sanders et al. 1988) proposed that galaxy interactions are the main triggering mechanism in the starbursts of dwarf and irregular galaxies. The interactions are not usual with nearby giant galaxies (Campos-Aguilar et al. 1993; Telles $\&$ Terlevich 1995), but they seem to be more frequent with low surface brightness galaxies (Taylor et al. 1996; Wilcots et al. 1996).

On the other hand, the gas-rich dwarf irregular galaxies could have formed by material ejected from the disks of the parent galaxies to the intergalactic medium by tidal forces (Okazaki \& Taniguchi 2000). The recycled objects formed in 
this way (the tidal dwarf galaxies, TDGs) give important clues to both age and past evolution of the galaxy (Duc \& Mirabel 1998). In some cases, star-forming TDGs are found at the end of long stellar tails in interacting systems (Weilbacher et al. 2003).

In one way or in another, the interactions seem to play a key role in the triggering of the star formation in dwarf galaxies. An increasing number of starburst galaxies show Wolf-Rayet (WR) features in their integrated spectra. The so-called Wolf-Rayet galaxies have a broad emission feature around $4650 \AA$ that usually consist of a blend of lines He II $\lambda 4686$ (the most important line), C III/C IV $\lambda 4650$ and N III $\lambda 4640$, and it indicates the presence of a substantial population of this sort of massive stars. The most massive OB stars became WR stars around 2 and $3 \mathrm{Myr}$ since their birth, and spend between 3 and 6 Myr in this phase (Maeder \& Meynet 1994). The short time in which the WR features can be observed makes these systems ideal objects to look for the probable interaction mechanism of the star formation. They also provide information about the age and properties of the starbursts. Studying a sample of WR galaxies, Méndez \& Esteban (2000) (hereinafter ME00) suggested that interactions with or between dwarf objects could be the main star formation triggering mechanism in dwarf galaxies. Once triggered, the evolution of a starburst depends on the details of its interaction with the galaxy environment. ME00 also remarked that the interacting and/or merging nature of the WR galaxies can only be detected when deep and high-resolution images and spectra are available.

Mkn 1087 (II Zw 23, UGC 3179) was firstly described by Zwicky (1971) as an elliptically shaped BCG with a very extended filament to the north and a less extended one to the south. These filaments were studied by Keel (1988) using optical imaging and spectroscopy and radio continuum mapping of the system. He found that the filaments were redshifted with respect to the central zones of the galaxy, suggesting that they are the result of neutral gas infalling from a very massive H I cloud in which Mkn 1087 is embedded. He proposed that Mkn 1087 is a galaxy in formation and suggested that the star formation process has been occurring continuously for the past $10^{9}$ year. He also remarked that it could be as a consequence of the interaction with a companion galaxy located $10^{\prime \prime}$ to the south of the Mkn 1087 nucleus (our knot \#7, see Sect. 3.1). Mkn 1087, which is at around $111 \mathrm{Mpc}$ (assuming $H_{0}=75 \mathrm{~km} \mathrm{~s}^{-1} \mathrm{Mpc}^{-1}$, at this distance $1^{\prime \prime}=538 \mathrm{pc}$ ), has a companion galaxy named KPG 103a, at $81 \mathrm{kpc}$ to the southwest and at the same redshift (Marzke et al. 1996). Malkan et al. (1998) obtained a HST WFPC2 image of Mkn 1087 showing that it is a spiral galaxy with dust lanes on one side of its major axis.

The presence of WR features in the integrated spectrum of Mkn 1087 was first investigated by Kunth \& Joubert (1985), who gave an upper limit to the flux of the blue WR bump around He II 14686 . Vacca \& Conti (1992) did not measure the He II $\lambda 4686$ emission line but reported an upper limit of 1600 to the total late-type nitrogen-rich WR stars (WNL) contained in the central region of Mkn 1087. Vaceli et al. (1997) performed a spectroscopical study of 46 galaxies with emission line spectra in where Mkn 1087 was included, and reported a detection of the He II $\lambda 4686$ emission line, giving its flux with an error less than $50 \%$. However, they did not give information about its line width, so the nebular or stellar origin can not be specified. As consequence, Schaerer et al. (1999) classified Mkn 1087 as a suspected Wolf-Rayet galaxy.

ME00 presented deep CCD UBV images of Mkn 1087, finding and characterizing several faint objects connected by bridges and tails with the main body of the galaxy. They used Leitherer \& Heckman (1995) population synthesis models to estimate the ages of the emission zones detected in their images, finding that some of these emission knots are around 5 Myr old. ME00 interpreted Mkn 1087 as a galaxy experiencing an interaction event with one or more of the neighboring objects. The main aim of this study is to perform a more complete observational analysis of these surrounding objects in order to establish their nature.

\section{Observations and data reduction}

\subsection{Optical imaging}

Optical $V$ images of Mkn 1087 were carried out on 2000 December 19 with CAFOS (Calar Alto Faint Object Spectrograph) in image mode at the Cassegrain focus of the $2.2 \mathrm{~m}$ telescope of the Centro Astronómico Hispano-Alemán (CAHA) at Calar Alto Observatory (Almería, Spain). A SITe CCD detector $(2048 \times 2048$ pixels $)$ with a pixel size of $24 \mu \mathrm{m}$ and spatial resolution of $0.53^{\prime \prime}$ pixel $^{-1}$ was used, as well as the standard Johnson $V$ filter. Three 1200 s exposures were added for each filter to obtain a good signal-to-noise and an appropriate removal of cosmic rays in the final images. The measured full width at half maximum $(F W H M)$ of the point-spread function (PSF) was approximately $1^{\prime \prime}$. Images were taken under photometric conditions, and the standard field 98-1119 of Landolt (1992) was used to perform the flux calibration.

Images in $B$ and $R$ filters were taken on 2004 January 20 at the $2.56 \mathrm{~m}$ Nordic Optical Telescope (NOT) at Roque de los Muchachos Observatory (La Palma, Canary Islands, Spain). We used the ALFOSC (Andalucia Faint Object Spectrograph and Camera) in image mode with a Loral/Lesser CCD detector $(2048 \times 2048$ pixels $)$ with a pixel size of $15 \mu \mathrm{m}$ and spatial resolution of $0.19^{\prime \prime}$ pixel $^{-1}$, and the standard Johnson filters. We obtained three $300 \mathrm{~s}$ exposures for $B$ filter and six $300 \mathrm{~s}$ exposures for $R$ filter, that were combined to obtain the final images with FWHM of the PSF of $0.8^{\prime \prime}$. The standard star SA97-284 (Landolt 1992) was used to flux calibrate the final images.

Twilight images of different zones of the sky (blank fields) were taken for each filter in order to perform the flat-field correction. All the reduction process (bias subtraction, flat-fielding and flux calibration) was done with IRAF $^{1}$ package following standard procedures. IRAF software was also used both to determine the $3 \sigma$ contours over the sky background level and to obtain the photometric values of the galaxies and the knots surrounding Mkn 1087.

We have also re-analysed the previous $U, B$ and $V$ images obtained by ME00 at $2.56 \mathrm{~m}$ NOT (Nordic Optical Telescope)

1 IRAF is distributed by NOAO which is operated by AURA Inc., under cooperative agreement with NSF. 


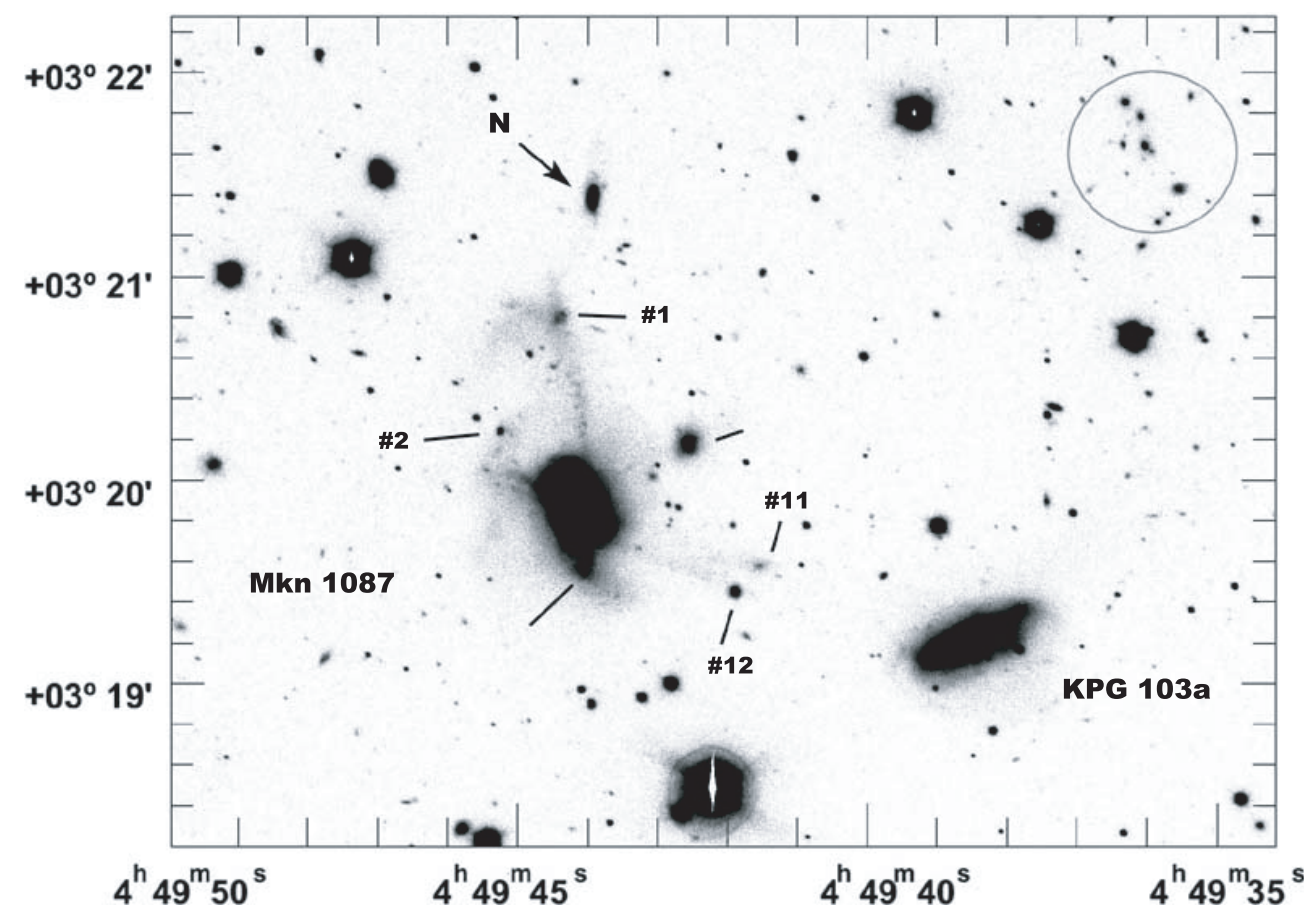

Fig. 1. Deep optical image of Mkn 1087 and its surrounding in $R$ filter. Mkn 1087, KPG 103a, the north companion galaxy (N) and the rest of the surrounding objects are labeled. The background cluster of galaxies is indicated with a circle.

on 1997 February 6. See ME00 for a detailed description of these observations.

\subsection{Near-infrared imaging}

We used the $1.55 \mathrm{~m}$ Carlos Sánchez Telescope (CST) at Teide Observatory (Tenerife, Canary Islands, Spain) to obtain the near-infrared images on 2002 September 24. We used CAIN camera $(256 \times 256$ pixels $)$ with a pixel size of $40 \mu \mathrm{m}$ and a spatial resolution of $1^{\prime \prime}$ pixel $^{-1}$ in the wide mode to obtain images in $J(1.2 \mu \mathrm{m}), H(1.6 \mu \mathrm{m})$ and $K_{\mathrm{s}}(2.18 \mu \mathrm{m})$ broad-band filters. We took 10 series of 6 consecutive individual $20 \mathrm{~s}$ exposures in $J$ filter, 10 series of 12 individual $10 \mathrm{~s}$ exposures in $H$ filter and 10 series of 12 exposures of $5 \mathrm{~s}$ duration in $K_{\mathrm{s}}$. Each series of exposures was made at slightly different positions to obtain a clean sky image. In this way, we obtained a single image of $20 \mathrm{~min}$ in $J$ and $H$ filters, and a single one of $10 \mathrm{~min}$ in $K_{\mathrm{s}}$ filter. We repeated this procedure two times for $J$ and $H$ and three times for $K_{\mathrm{s}}$, combining these images in a definitive one for each filter to increase the signal-to-noise ratio. The FWHM of the PSFs of these final images was $2.8^{\prime \prime}$, $2.6^{\prime \prime}$ and $2.5^{\prime \prime}$ for $J, H$ and $K_{\mathrm{s}}$, respectively. Bright and dark dome flat-field exposures were taken for each filter that were combined to obtain a good flat-field image. The standard stars As01 and As11 (Hunt et al. 1998) were used for flux calibrate the images.

\subsection{Intermediate-resolution spectroscopy}

We have obtained long-slit intermediate-resolution spectroscopy in five slit positions centered on the nucleus of the galaxy and covering most of the surrounding objects. We have used the $4.2 \mathrm{~m}$ William Herschel Telescope (WHT) and the $2.5 \mathrm{~m}$ Isaac Newton Telescope (INT), both at Roque de los Muchachos Observatory (La Palma, Canary Islands).

INT spectroscopy was carried out on 2000 December 28 and 29 with the IDS spectrograph at the Cassegrain focus with the $235 \mathrm{~mm}$ camera. An EEV CCD with a configuration of $2148 \times 4200$ pixels of $13.5 \mu \mathrm{m}$ was used. The slit was $2.8^{\prime}$ long and $1^{\prime \prime}$ wide. The grating R400V was used, with a dispersion of $104.5 \AA \mathrm{mm}^{-1}$ and effective spectral resolution of $1.40 \AA$. Each individual spectrum covers from 3200 to $7700 \AA$. The spatial resolution is $0.40^{\prime \prime} \mathrm{pixel}^{-1}$. Four slit positions of Mkn 1087 were observed at different position angles, which were chosen in order to cover different morphological zones. Three or four $20 \mathrm{~min}$ exposures were taken and combined to obtain good signal-to-noise and an appropriate removal of cosmic rays in the final spectra for each slit position. Comparison lamp exposures of $\mathrm{CuAr}$ were taken after each set of spectra. The absolute flux calibration was achieved by observations of the standard stars Feige 56, Hiltner 600 and Feige 110 (Massey et al. 1988).

An additional slit position of Mkn 1087 was obtained using $4.2 \mathrm{~m}$ WHT. It was carried out on 2000 December 31 with the ISIS spectrograph at the Cassegrain focus. Two different CCDs were used at the blue and red arms of the spectrograph: an EEV CCD with a configuration of $4096 \times 2048$ pixels of $13 \mu \mathrm{m}$ in the blue arm and a TEK with $1024 \times 1024$ of $24 \mu \mathrm{m}$ in the red arm. The dichroic used to separate the blue and red beams was set at $5400 \AA$. The slit was $3.7^{\prime}$ long and $1^{\prime \prime}$ wide. Two gratings were used, the R600B in the blue arm and the $\mathrm{R} 316 \mathrm{R}$ in the red arm. These gratings give reciprocal dispersion of 33 and $66 \AA \mathrm{mm}^{-1}$, and effective spectral resolutions of 2.0 and $3.9 \AA$ for the blue and red arms, respectively. The blue 
spectra cover from 3600 to $5200 \AA$ and the red ones from 5400 to $6800 \AA$. The spatial resolution is $0.20^{\prime \prime}$ pixel $^{-1}$ in the blue and $0.35^{\prime \prime}$ pixel $^{-1}$ in the red. We obtained three 30 min exposures in each arm that were combined to obtain the final blue and red spectra for this slit position. Comparison lamp exposures of $\mathrm{CuAr}$ for the blue arm and $\mathrm{CuNe}$ for the red one were taken after each set of spectra. The absolute flux calibration was achieved by observations of the standard stars G191, B2B and Feige 34 (Massey et al. 1988). WHT observations were made at air masses around 1.1. INT observations were made at air masses between 1.1 and 1.3. Consequently, no correction was made for atmospheric differential refraction.

All the CCD frames for spectroscopy were reduced using standard IRAF procedures to perform bias correction, flatfielding, cosmic-ray rejection, wavelength and flux calibration, and sky subtraction. The correction for atmospheric extinction was performed using an average curve for the continuous atmospheric extinction at Roque de los Muchachos Observatory. For each two-dimensional spectra several apertures were defined along the spatial direction to extract the final one-dimensional spectra of each knot, centering each aperture at its brightest point. For WHT observations, identical apertures were defined on the "blue" and "red" frames. Small two-dimensional distortions were corrected fitting the maxima of [O II] $\lambda \lambda 3726,3729$ doublet and $\mathrm{H} \alpha$ in INT frames, using also $\mathrm{H} \beta$ and continuum emission for WHT ones.

The journal of all the imaging and spectroscopical observations can be found in Table 1 .

\section{Results}

\subsection{Optical and near-infrared imaging}

The deep CAHA $V$ image of the system was presented in Esteban et al. (2003), showing the main non-stellar objects surrounding Mkn 1087 found by ME00. We designated the objects following those authors. Several bridges can be observed connecting the main body of Mkn 1087 with the objects \#1, \#2, $\# 3$, \#11 and \#12, the last two ones located in the direction of the nearby galaxy KPG 103a. Knot \#7 seems to be an intense star-formation zone off-center Mkn 1087.

In order to obtain a deeper image, we carried out new observations of the system in $R$ broad-band filter. We present this image in Fig. 1. The bridges commented before are now clearly detected, especially the ones that end at \#11 and \#12. The bright bridge connecting Mkn 1087 with \#1 is apparently splitted in several small knots. The north companion object seems to be clearly elliptical and shows a very weak plume in the direction to knot \#1. Although bridges between the two brightest galaxies are not detected, a long very weak plume can be observed at the east of KPG 103a. Another diffuse long plume is detected at the west. It is important to note the high number of (apparently) background non-stellar objects (presumably galaxies) that are detected in the new deep $R$ image (we have estimated around 60 candidates in the entire final $R$ frame). They are uniformly distributed in the field of view of the image (about $6^{\prime}$ ) and are most probably background galaxies. We also want to remark the cluster of galaxies found at the

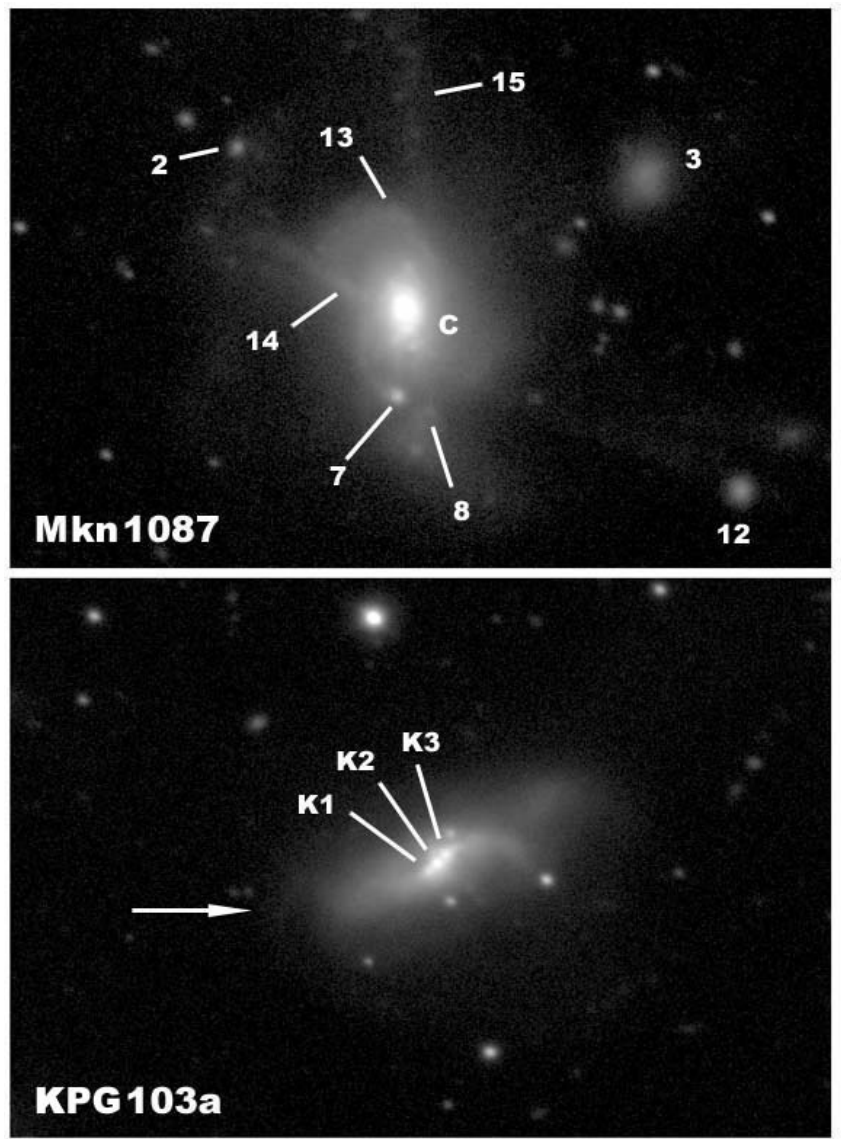

Fig. 2. Detailed images in $R$ band of Mkn 1087 (up) and KPG 103a (bottom). The brightest knots inside both galaxies are labeled, as well as the external objects \#2, \#3 and \#12. A weak plume at the east of KPG 103a, that curves toward the south, is also barely observed (it is indicated by an arrow).

upper right corner of the image, with central position around $\alpha=04^{\mathrm{h}} 49^{\mathrm{min}} 35^{\mathrm{s}}, \delta=+03^{\circ} 21^{\prime} 04^{\prime \prime}$. It is not catalogued in the NASA/IPAC Extragalactic Database (NED). We analyze it in Sect. 4.7.

Some important structures inside the main galaxies are now clearly distinguished. In Fig. 2 we show the central part of Mkn 1087 (up) and KPG 103a (down). The center of Mkn 1087, C (knot \#5 following ME00), is the brightest knot of the system. Mkn 1087 reveals two spiral arms or structures: an upper arm connecting with the bridge towards knot \#2 and a bottom arm that seems to end in knot \#7. Furthermore, some new knots are now detected inside the main body of both galaxies. Especially intense are the three bright knots K1, K2 and K3, that are resolved in the center of KPG 103a. They show blue colors (see Table 2) that are representative of young population, suggesting that these knots are star-formation areas in the nucleus of KPG 103a. The WFPC2 HST image of Mkn 1087 presented by Malkan et al. (1998) clearly shows knots C, \#7,\#8, \#13 and \#14 and the arms of the spiral disk we have distinguished in our NOT images. The HST image suggests that object \#2 could be a background object that is coincident with the eastern bridge of Mkn 1087, although this cannot be confirmed without a spectrum of the object. However, this 
Table 1. Summary of observations.

\begin{tabular}{ccccccccc}
\hline \hline Observations & Telescope & Date & $\begin{array}{c}\text { Exp. time } \\
(\mathrm{s})\end{array}$ & $\begin{array}{c}\text { Spatial } \\
\left({ }^{\prime \prime} \text { pix }\right.\end{array}$
\end{tabular}

Table 2. Results of optical and near-infrared aperture photometry in Mkn 1087 and KPG 103a knots.

\begin{tabular}{|c|c|c|c|c|c|c|c|c|c|c|}
\hline Knot & $E(B-V)$ & $m_{\mathrm{B}}$ & $m_{\mathrm{B}}{ }^{0}$ & $m_{\mathrm{J}}^{0}$ & $(U-B)_{0}$ & $(B-V)_{0}$ & $(V-R)_{0}$ & $(V-J)_{0}$ & $(J-H)_{0}$ & $(H-K \mathrm{~s})_{0}$ \\
\hline Mkn 1087 & $0.17^{a}$ & 13.78 & $13.08 \pm 0.04$ & $12.39 \pm 0.04$ & $-0.41 \pm 0.08^{b}$ & $0.17 \pm 0.08$ & $0.20 \pm 0.08$ & $0.52 \pm 0.06$ & $0.20 \pm 0.06$ & $0.16 \pm 0.06$ \\
\hline $\mathrm{C}$ & 0.14 & 15.19 & $14.62 \pm 0.03$ & $14.33 \pm 0.05$ & $-0.57 \pm 0.06^{b}$ & $-0.02 \pm 0.06$ & $0.11 \pm 0.06$ & $0.31 \pm 0.10$ & $0.15 \pm 0.10$ & $0.15 \pm 0.10$ \\
\hline $\mathrm{N}$ & 0.10 & 17.58 & $17.17 \pm 0.03$ & $17.01 \pm 0.06$ & $\ldots$ & $-0.05 \pm 0.06$ & $0.14 \pm 0.10$ & $0.21 \pm 0.08$ & $0.18 \pm 0.08$ & $0.13 \pm 0.08$ \\
\hline$\# 1$ & $0.07^{c}$ & 19.18 & $18.89 \pm 0.05$ & $>18.7$ & $-0.75 \pm 0.15^{b}$ & $-0.01 \pm 0.08$ & $0.10 \pm 0.08$ & $<0.2$ & $\ldots$ & $\ldots$ \\
\hline$\# 2$ & $0.07^{c}$ & 20.01 & $19.72 \pm 0.05$ & $>18.7$ & $-0.78 \pm 0.15^{b}$ & $0.05 \pm 0.08$ & $0.12 \pm 0.08$ & $<1$ & $\ldots$ & $\ldots$ \\
\hline$\# 3$ & $0.07^{c}$ & 18.32 & $18.03 \pm 0.03$ & $17.28 \pm 0.06$ & $0.08 \pm 0.30^{b}$ & $0.11 \pm 0.06$ & $0.26 \pm 0.06$ & $0.64 \pm 0.10$ & $0.5 \pm 0.2$ & $\ldots$ \\
\hline \#7 & 0.16 & 18.29 & $17.63 \pm 0.04$ & $\ldots$ & $-0.87 \pm 0.08^{b}$ & $-0.04 \pm 0.08$ & $0.03 \pm 0.08$ & $\ldots$ & $\ldots$ & $\ldots$ \\
\hline$\# 8$ & 0.08 & 19.10 & $18.77 \pm 0.05$ & $\ldots$ & $-0.27 \pm 0.12^{b}$ & $0.07 \pm 0.10$ & $0.17 \pm 0.08$ & $\ldots$ & $\ldots$ & $\ldots$ \\
\hline \#11 & $0.07^{c}$ & 20.69 & $20.40 \pm 0.05$ & $\ldots$ & $\ldots$ & $0.35 \pm 0.10$ & $0.08 \pm 0.10$ & $\ldots$ & $\ldots$ & $\ldots$ \\
\hline$\# 12$ & $0.07^{c}$ & 19.61 & $19.32 \pm 0.05$ & $18.5 \pm 0.1$ & $-0.1 \pm 0.3$ & $0.31 \pm 0.09$ & $0.24 \pm 0.08$ & $0.55 \pm 0.15$ & $0.4 \pm 0.2$ & $\ldots$ \\
\hline$\# 13$ & 0.40 & 19.25 & $17.61 \pm 0.05$ & $\ldots$ & $-0.64 \pm 0.12$ & $0.01 \pm 0.09$ & $0.04 \pm 0.08$ & $\ldots$ & $\ldots$ & $\cdots$ \\
\hline$\# 14$ & 0.07 & 18.45 & $18.16 \pm 0.05$ & $\ldots$ & $-0.59 \pm 0.12$ & $0.28 \pm 0.10$ & $0.23 \pm 0.10$ & $\ldots$ & $\ldots$ & $\ldots$ \\
\hline$\# 15$ & 0.19 & 20.90 & $20.12 \pm 0.08$ & $\ldots$ & $-0.6 \pm 0.3$ & $0.22 \pm 0.12$ & $0.23 \pm 0.10$ & $\ldots$ & $\ldots$ & $\ldots$ \\
\hline KPG 103a & $0.07^{c}$ & 14.05 & $13.77 \pm 0.04$ & $\ldots$ & $0.17 \pm 0.08^{b}$ & $0.18 \pm 0.06$ & $0.27 \pm 0.06$ &.. & $\ldots$ & $\ldots$ \\
\hline K1 & $0.07^{c}$ & 17.55 & $17.26 \pm 0.05$ & $\ldots$ & $-0.40 \pm 0.10$ & $0.18 \pm 0.10$ & $0.20 \pm 0.08$ & $\ldots$ & $\ldots$ & $\ldots$ \\
\hline K2 & $0.07^{c}$ & 17.10 & $16.81 \pm 0.04$ & $\ldots$ & $-0.49 \pm 0.08$ & $0.12 \pm 0.06$ & $0.11 \pm 0.06$ & $\ldots$ & $\ldots$ & $\ldots$ \\
\hline K3 & $0.07^{c}$ & 17.41 & $17.12 \pm 0.04$ & $\ldots$ & $-0.39 \pm 0.08$ & $0.19 \pm 0.06$ & $0.33 \pm 0.06$ & $\ldots$ & $\ldots$ & $\ldots$ \\
\hline
\end{tabular}

${ }^{a}$ Average of the values obtained for C, \#7, \#8, \#13, \#14 and \#15.

${ }^{b}$ Adopting the $m_{U}$ values from ME00.

${ }^{c}$ Galactic reddening adopted from Schlegel et al. (1998), $E(B-V)=0.07$.

knot shows very blue colors, $(U-B)=-0.78$, similar to other knots around Mkn 1087, so we prefer to consider knot \#2 in our study.

In Fig. 3 we show the logarithmic contour map of Mkn 1087 and its surroundings in filters $B$ and $J$. We clearly detect the north companion object in all NIR images. However, the knots \#3 and \#12 are only detected in $J$ and $H$ filters. Knots \#1 and \#2 are barely detected in the $J$ image. We did not observe the galaxy KPG 103a in NIR.

ME00 performed aperture photometry of the knots of the system in $U, B$ and $V$ filters. We have improved their analysis performing the aperture photometry of the companion object in our new $B, V$ and $R$ images. We have determined the area for which we have integrated the flux using the $3 \sigma$ level isophote over the average sky level in the $B$ image. The same integration area was then used for $V$ and $R$ filters. We have also measured the rest of the knots in the system, finding similar values to those obtained by ME00. In addition, we have determined the photometric $U, B$ and $V$ values of some new interesting areas (\#13, \#14 and \#15) because they correspond to localized bright nebula emission in the disk of Mkn 1087 as our spectroscopy reveals (see next section), they probably correspond to giant H II regions. Finally, we have analyzed the NIR images to obtain the photometric $J, H$ and $K_{\mathrm{s}}$ values of the detected 


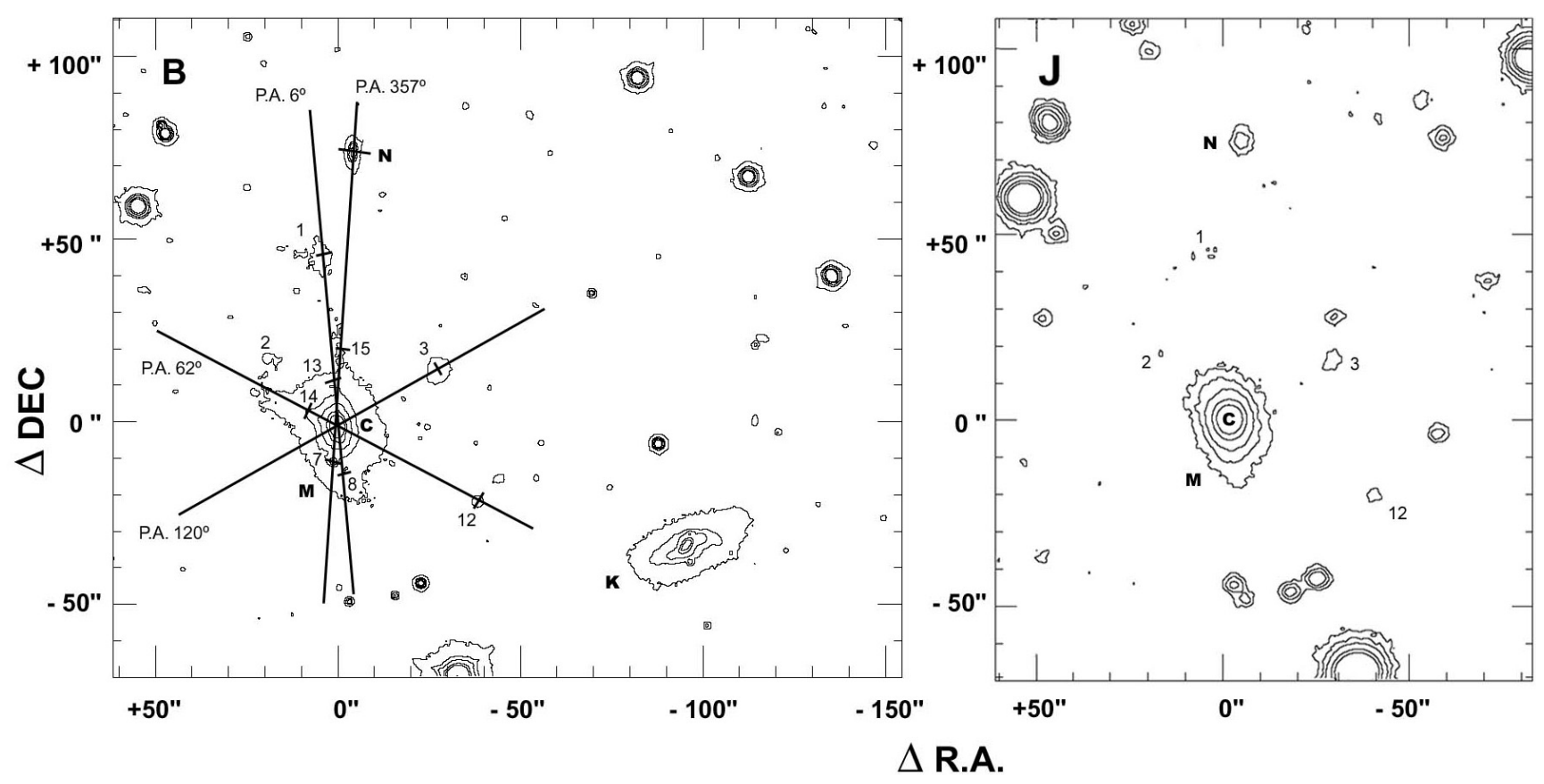

Fig. 3. Logarithmic contour maps in $B$ and $J$ filters of Mkn 1087. M indicates the main body of Mkn 1087, C the center of it, K the nearby galaxy KPG 103a and N the north companion galaxy. The contours are $3 \sigma, 10 \sigma, 20 \sigma, 50 \sigma$ and $100 \sigma$ over the sky level for both maps. The slit positions used for spectroscopy are also shown over the $B$ image, indicating with ticks the center of the different zones from which we have extracted the one-dimensional spectra.

objects. The integration area in $J$ filter (defined as the $3 \sigma$ level isophote over the sky level) was used for each NIR filter. We also estimated a lower limit for $m_{J}$ in knots \#1 and \#2. All these new optical and NIR data are shown in Table 2 . We only give upper limits to the $J$ magnitudes for knots \#1 and \#2. Hunter \& Gallagher (1985) performed $J H K$ photometry within $23^{\prime \prime}$ aperture and found $J-H=0.65, H-K=0.28$ and $V-J=1.76$, suggesting a dominant contribution of old populations at these wavelengths.

The reddening constant, $C(\mathrm{H} \beta)$ - obtained from the absorption-corrected intensities of H I Balmer lines in our optical spectra of each member - was used to correct the photometric data for the extinction by interstellar dust. We describe the method used to obtain $C(\mathrm{H} \beta)$ from the spectra in the next section. The relation obtained by Kaler \& Lutz (1985) between $C(\mathrm{H} \beta)$ and the extinction in $V$ filter $\left(A_{V}\right)$ was used $\left[A_{V} \sim 2 C(\mathrm{H} \beta)\right]$, and a standard ratio of $R_{V}=A_{V} / E(B-V)=$ 3.1 was assumed. Calzetti et al. (2000) propose a starburst reddening curve with $R_{V}=4.05 \pm 0.80$. If we use this curve for our objects, the $B$ magnitudes would be between 0.08 and 0.24 brighter than the ones we have calculated assuming $R_{V}=3.1$. We would also obtain bluer colors, but the differences are smaller than the photometric uncertainties. The choice between the two reddening curves implies only slight changes in the results and does not change our conclusions. The data in $U, B, R, J, H$ and $K_{\mathrm{s}}$ filters were corrected using the Rieke \& Lebofsky (1985) extinction correction law for $A_{U}$, $A_{B}, A_{R}, A_{J}, A_{H}$ and $A_{K \mathrm{~s}}$. For the main body of Mkn 1087 we have assumed an average extinction using the objects $C$, \#7, \#8, \#13, \#14 and \#15, that are the knots inside Mkn 1087 for which we have spectra. Schlegel et al. (1998) presented an all-sky map of infrared dust emission, determining a color excess of $E(B-V)=0.07$ (assuming $R_{V}=3.1$ ) at the Galactic longitude and latitude of Mkn 1087. This value should be considered as a lower limit to the real one. We have assumed only this Galactic contribution to the reddening in the direction of Mkn 1087 for the more external objects (\#1, \#2, \#3 and \#12) and the galaxy KPG 103a because we have no determination of $C(\mathrm{H} \beta)$ for it. The final $E(B-V)$ adopted for each individual object is also shown in Table 2. We have determined the errors for the photometry considering the FWHM of the PSF, sky level and flux calibration for each frame. The error in $V-J$ color due to the differences in the shape and size between the optical and the NIR integrated areas is small when compared with the other uncertainties.

\subsection{Intermediate resolution spectra}

We have observed five slit positions in Mkn 1087 in order to obtain the spectra of the most interesting zones. In Fig. 3 we show the four different position angles (PA) obtained using the INT over the $B$ contour map, as well as the center of each extracted 1-D spectrum. All four slit positions cover the central zone, C, of Mkn 1087 and were combined to obtain an individual high signal-to-noise spectrum of this zone. The slit with PA $6^{\circ}$ covers knots \#1, \#8 and the new bright emission area \#13. The slit with PA $62^{\circ}$ passes through knots \#12 (that does not show nebular emission) and the new object \#14. Knot \#3 was observed with PA $120^{\circ}$, but it only reveals a faint nebular emission. The new companion galaxy and the intense knot \#7 were observed using the slit position with PA $357^{\circ}$, that also shows the new nebular zone \#15. The single slit position observed using WHT 
Table 3. General spectroscopic properties of the knots.

\begin{tabular}{|c|c|c|c|c|c|c|c|c|c|c|c|c|}
\hline Knot & C WHT & C INT & N WHT & N INT & $7 \mathrm{WHT}$ & \#7 INT & \#\#1 & \#33 & \#8 & $\# 13$ & \#\#14 & $\# 15$ \\
\hline$-M_{B}$ & 20.61 & 20.61 & 18.06 & 18.06 & 17.60 & 17.60 & 16.34 & 17.20 & 16.46 & 17.62 & 17.07 & 15.11 \\
\hline \pm & 0.03 & 0.03 & 0.03 & 0.03 & 0.04 & 0.04 & 0.05 & 0.03 & 0.05 & 0.05 & 0.05 & 0.08 \\
\hline Slit $\left(" \times 1^{\prime \prime}\right)$ & 5.4 & 12 & 3.6 & 8.4 & 6.0 & 3.6 & 5.6 & 4.0 & 5.2 & 7.2 & 5.6 & 8.0 \\
\hline Distance $\left({ }^{\prime \prime}\right)^{a}$ & 0 & 0 & 77.0 & 77.0 & 9.8 & 10.0 & 47.6 & 35.2 & 10.9 & 10.8 & 8.0 & 19.2 \\
\hline$C(\mathrm{H} \beta)^{b}$ & 0.17 & 0.25 & 0.17 & 0.12 & 0.24 & 0.25 & 0.10 & 0.10 & 0.12 & 0.62 & 0.11 & 0.29 \\
\hline \pm & 0.02 & 0.03 & 0.03 & 0.03 & 0.03 & 0.03 & 0.03 & 0.03 & 0.03 & 0.12 & 0.04 & 0.05 \\
\hline$W_{\mathrm{abs}}^{b, c}$ & 1.7 & 2.4 & 0.2 & 0.9 & 2.9 & 1.5 & $\ldots$ & $\ldots$ & 1.1 & 2 & 5.6 & 2 \\
\hline \pm & 0.1 & 0.3 & 0.2 & 0.2 & 0.5 & 0.3 & $\ldots$ & $\ldots$ & 0.3 & $\ldots$ & 1.7 & ... \\
\hline$T_{\mathrm{e}}[\mathrm{O} \mathrm{III}]^{d}$ & 0.71 & 0.74 & 1.09 & 1.13 & 0.77 & 0.78 & 0.82 & 0.76 & 0.88 & 0.82 & 0.86 & 0.81 \\
\hline$T_{\mathrm{e}}[\mathrm{O} \mathrm{III}]^{e}$ & 0.80 & 0.82 & 1.06 & 1.09 & 0.84 & 0.84 & 0.88 & 0.83 & 0.92 & 0.87 & 0.90 & 0.87 \\
\hline$N_{\mathrm{e}}\left(\mathrm{cm}^{-3}\right)$ & 220 & 170 & 115 & 150 & 120 & 210 & $\ldots$ & $\ldots$ & 150 & 120 & $<100$ & $<100$ \\
\hline$\Delta v_{r}^{f}$ & 0 & 0 & +117 & +110 & +118 & +122 & -63 & +8 & +125 & -85 & -70 & -75 \\
\hline \pm & $\ldots$ & $\ldots$ & 15 & 27 & 15 & 27 & 27 & 80 & 27 & 27 & 35 & 35 \\
\hline$F(\mathrm{H} \beta)^{g}$ & 133 & 184 & 4.58 & 11.3 & 16.6 & 1.76 & 3.05: & 1.58: & 6.03 & 1.92 & 8.46 & 0.94: \\
\hline \pm & 4 & 6 & 0.16 & 0.6 & 0.7 & 0.09 & $\ldots$ & $\ldots$ & 0.37 & 0.26 & 0.62 & $\ldots$ \\
\hline$W(\mathrm{H} \alpha)^{c}$ & 87.4 & 87.3 & 53.6 & 66.5 & 330 & 256 & 110 & 20: & 72.0 & 30.9 & 140 & 39.7 \\
\hline \pm & 2.6 & 3.4 & 2.1 & 8.2 & 25 & 28 & 25 & $\ldots$ & 4.5 & 2.8 & 20 & 9.5 \\
\hline$W(\mathrm{H} \beta)^{c}$ & 22.3 & 22.9 & 25.0 & 27.4 & 62.3 & 66 & $\ldots$ & $\ldots$ & 16.6 & 5.6 & 52 & 8.0: \\
\hline \pm & 0.9 & 2.1 & 1.6 & 2.4 & 5.4 & 12 & $\ldots$ & $\ldots$ & 2.6 & 1.3 & 8 & $\ldots$ \\
\hline$W(\mathrm{H} \gamma)^{c}$ & 8.1 & 6.6 & 8.2 & 5.3 & 34.3 & 20.2 & $\ldots$ & $\ldots$ & 8.3 & $\ldots$ & 18 & $\ldots$ \\
\hline \pm & 0.4 & 0.6 & 0.9 & 0.5 & 4.9 & 2.2 & $\ldots$ & $\ldots$ & 1.6 & $\ldots$ & 5 & $\ldots$ \\
\hline$W([\mathrm{O} \mathrm{III}])^{c}$ & 16.7 & 20.5 & 144 & 46.6 & 116 & 63.2 & $\ldots$ & $\ldots$ & 25.7 & 6.8 & 42.0 & 7.8: \\
\hline \pm & 0.6 & 0.9 & 18 & 3.7 & 9 & 3.6 & $\ldots$ & $\ldots$ & 2.8 & 1.2 & 3.6 & $\ldots$ \\
\hline $\mathrm{Age}^{h}$ & 6.0 & 6.0 & 7 & 6 & 5.0 & 4.5 & 6 & 150 & 6 & 8.5 & 5 & 8.0 \\
\hline \pm & 0.5 & 0.5 & 1 & 1 & 0.5 & 0.5 & 2 & 30 & 1 & 1.5 & 1 & 1.5 \\
\hline
\end{tabular}

${ }^{a}$ Distance from the center of Mkn 1087.

${ }^{b} C(\mathrm{H} \beta)$ and $W_{\text {abs }}$ calculated iteratively. See Sect. 3.2 for more details.

${ }^{c}$ In $\AA$.

${ }^{d}$ In units of $10^{4} \mathrm{~K}$, calculated using empirical calibrations of Pilyugin (2001).

${ }^{e}$ In units of $10^{4} \mathrm{~K}, T_{\mathrm{e}}[\mathrm{OII}]$ calculated from empirical calibration and the relation given by Garnett (1992).

${ }^{f}$ Radial velocity with respect to the center of Mkn 1087, in $\mathrm{km} \mathrm{s}^{-1}$.

${ }^{g}$ In units of $10^{-16} \mathrm{erg} \mathrm{s}^{-1} \mathrm{~cm}^{-2}$.

${ }^{h}$ Estimated ages using our spectroscopical and photometric data, in Myr. See Sect. 4.1 for more details.

has a PA $358^{\circ}$ and is not represented in Fig. 3 (it is similar to the PA $357^{\circ}$ shown in the figure) and covers the center of Mkn 1087, the companion galaxy and knot \#7. The distance to the center of Mkn 1087 and the size of each extracted 1-D spectrum is indicated in Table 3. Note that we have observed six different areas at different radii along the main body of Mkn 1087, two of them (C and \#7) observed both with INT and WHT.

The wavelength and flux calibrated spectra of the center of Mkn 1087, the north companion and knot \#7 are shown in Fig. 4. The spectrum of the center of Mkn 1087 shows a high continuum level and an intense underlying stellar absorption in $\mathrm{H}$ I Balmer lines. Absorption wings are clearly resolved for $\mathrm{H} \beta$, $\mathrm{H} \gamma$ and $\mathrm{H} \delta$. The high continuum level and these absorption features can be interpreted as the product of a substantial population of stars with ages larger than $10 \mathrm{Myr}$ (see Sect. 4.1). The spectra of the other regions or knots show an almost absent continuum level with no evident absorption features, characteristic of a $\mathrm{H}$ II region-dominated zone.

Fluxes and equivalent widths of the emission lines for each spectrum were measured using the SPLOT routine of the IRAF package. This task integrates all the flux in the line between two given limits and over a fitted local continuum. We also used Starlink DIPSO (Howarth \& Murray 1990) software for the Gaussian fitting of profiles affected by line-blending (mainly, the [O II] $\lambda \lambda 3726,3729$ doublet in WHT spectra) and to obtain the best values for the fluxes of H I Balmer lines. For each single or multiple Gaussian fit, DIPSO gives the fit parameter (radial velocity centroid, Gaussian sigma, $F W H M$, etc.) and their associated statistical errors. To correct the observed line intensities for interstellar reddening we have used the reddening constant, $C(\mathrm{H} \beta)$, derived from the fluxes of H I Balmer lines. However, we must also take into account the effect of 


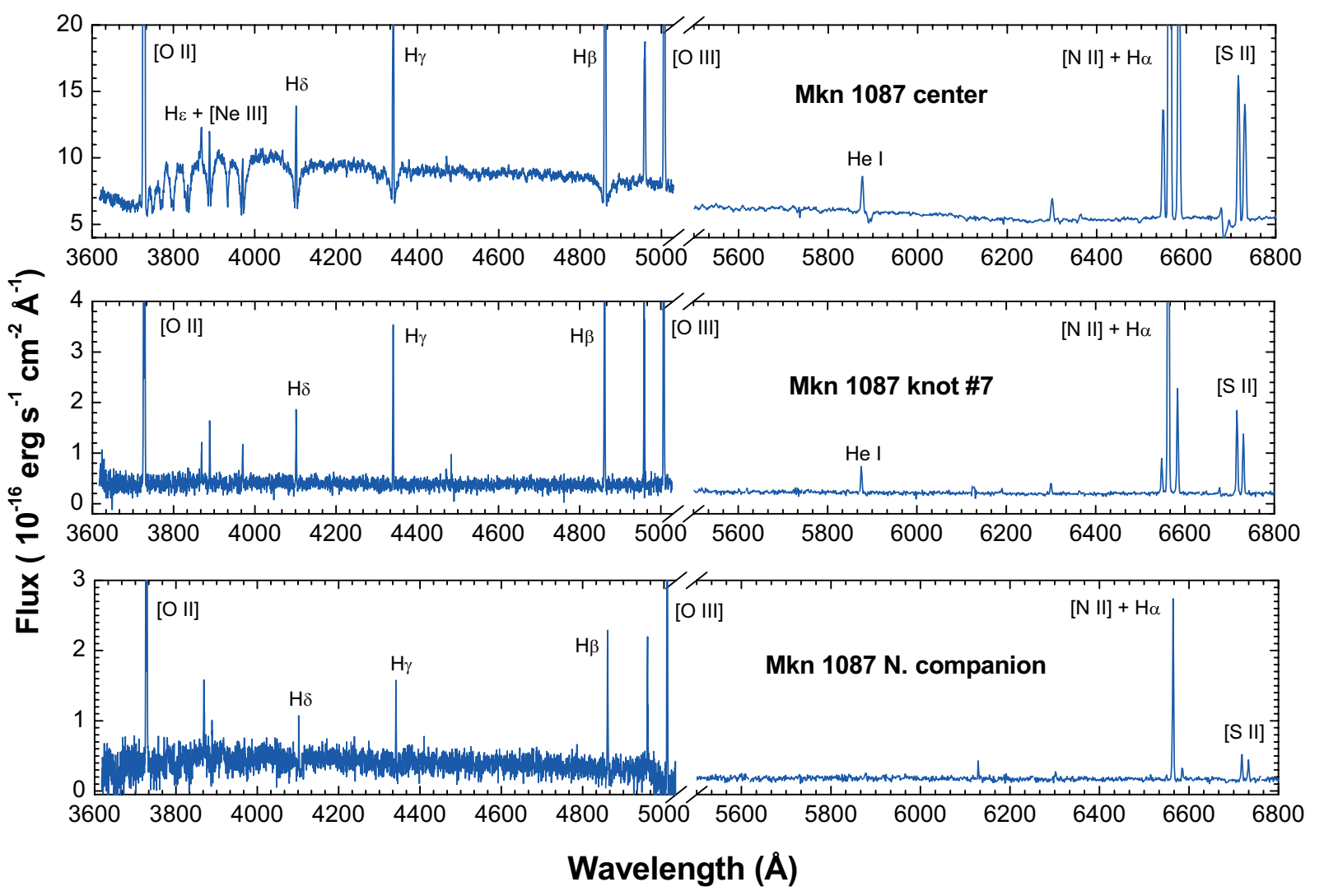

Fig. 4. ISIS WHT spectra of the center of Mkn 1087, the intense knot \#7 and its north companion object. The most important emission lines are labeled in the upper spectrum. All the spectra have been scaled down in flux in order to distinguish the faint lines. Note the high continuum level and the H I absorptions due to the underlying stellar population in the spectrum of the center of Mkn 1087. All spectra are redshift-corrected.

the underlying stellar absorption in the hydrogen lines. Their equivalent widths, $W_{\text {abs }}$, are usually considered, but their values are uncertain because they also depend on the age of burst (Oloffsson 1995). We have performed an iterative procedure to derive both, $C(\mathrm{H} \beta)$ and $W_{\mathrm{abs}}$, for each spectrum, assuming that the equivalent width of the absorption lines is the same for all the Balmer lines and using the relation given by Mazzarrella \& Boronson (1993) to the absorption correction together with the Whitford (1958) law:

$C(\mathrm{H} \beta)=\frac{1}{f(\lambda)} \log \left[\frac{\frac{I(\lambda)}{I(\mathrm{H} \beta)} \times\left(1+\frac{W_{\mathrm{abs}}}{W_{\mathrm{H} \beta}}\right)}{\frac{F(\lambda)}{F(\mathrm{H} \beta)} \times\left(1+\frac{W_{\mathrm{abs}}}{W_{\lambda}}\right)}\right]$

for each detected hydrogen Balmer line. In this equation, $F(\lambda)$ and $I(\lambda)$ are the observed and the theoretical fluxes (not affected by reddening or absorption), whereas $W_{\mathrm{abs}}, W_{\lambda}$ and $W_{\mathrm{H} \beta}$ are the equivalent widths of the underlying stellar absorption, the considered Balmer line and $\mathrm{H} \beta$, respectively. $f(\lambda)$ is the reddening curve normalized to $\mathrm{H} \beta$. We have considered the theoretical ratios between each pair of H I Balmer lines expected for case B recombination given by Brocklehurst (1971) and appropriated electron temperatures between 6000 and $10000 \mathrm{~K}$ and electron densities around $100 \mathrm{~cm}^{-3}$. The $C(\mathrm{H} \beta)$ and $W_{\mathrm{abs}}$ that provide the best match between the corrected and the theoretical line ratios for each knot are shown in Table 3. We have assumed $W_{\text {abs }}=2 \AA$ in knots \#13 and \#15 because we only detected the two brightest H I Balmer lines. For \#1 and \#3 we have not corrected for $W_{\text {abs }}$ due to their faintness absorption.

Finally, all the emission lines detected were corrected for reddening using the Whitford (1958) law and the corresponding value of $C(\mathrm{H} \beta)$. The $\mathrm{H} \beta$ line flux, $F(\mathrm{H} \beta)$ (corrected by reddening and underlying stellar absorption) and the equivalent width of several lines $(\mathrm{H} \alpha, \mathrm{H} \beta, \mathrm{H} \gamma$ and [O III] $)$ are given in Table 3, while the reddening-corrected line intensity ratios relative to $\mathrm{H} \beta$ of each observed object are given in Tables 4 and 5 . We have estimated the uncertainties in the line intensities following the equation given by Castellanos (2000). Colons indicate uncertainties of the order or greater than $40 \%$.

\subsubsection{Physical conditions of the ionized gas}

The 1-D spectra were used to study the physical conditions and chemical abundances of the ionized gas. Unfortunately, the auroral [O III] $\lambda 4363$ line is not detected or well measured, so we can not derive the electron temperature of the ionized gas. Although [O III] $\lambda 4363$ seems to be detected in the spectrum of the center of Mkn 1087, the broad absorption wing in $\mathrm{H} \gamma$ does not permit a proper measurement of this weak emission line. Consequently, the oxygen abundance for the different knots in Mkn 1087 was derived using the so-called empirical calibrations (see next section). The electron temperatures have been estimated from the $T([\mathrm{O} \mathrm{III}])$ and $T([\mathrm{O} \mathrm{II}])$ pairs that reproduce the total oxygen abundance obtained applying the Pilyugin (2001) empirical method (see next section). As Pilyugin, we 
Table 4. Dereddened line intensity ratios with respect to $I(\mathrm{H} \beta)=100$ for the three brightest star-forming bursts observed in Mkn 1087: the center of Mkn 1087 (C), the north companion galaxy (N) and the intense burst \#7. All have been observed both with $4.2 \mathrm{~m}$ WHT and $2.5 \mathrm{~m}$ INT. Correction for underlying stellar absorption in $\mathrm{H}$ I Balmer lines has been also applied.

\begin{tabular}{|c|c|c|c|c|c|c|c|}
\hline Line & $f(\lambda)$ & C WHT & C INT & N WHT & N INT & \#7 WHT & \#7 INT \\
\hline 3726 [O II] & 0.27 & $206 \pm 11^{a}$ & $226 \pm 13^{a}$ & $155 \pm 12$ & $325 \pm 26^{a}$ & $85.5 \pm 7.1$ & $212 \pm 20^{a}$ \\
\hline 3729 [O II] & 0.27 & $\ldots$ & $\ldots$ & $231 \pm 14$ & $\ldots$ & $106 \pm 8$ & $\ldots$ \\
\hline 3869 [Ne III] & 0.23 & $8.3 \pm 2.9$ & $8.7 \pm 3.3$ & $43.4 \pm 6.9$ & $37.8 \pm 9.1$ & $9.9 \pm 3.6$ & $17.1 \pm 6.2$ \\
\hline $3889 \mathrm{He} \mathrm{I}+\mathrm{H} 8$ & 0.22 & $13.8 \pm 4.3$ & $7.6 \pm 2.6$ & $\ldots$ & $\ldots$ & $15.0 \pm 4.3$ & $15.6 \pm 4.6$ \\
\hline 3968 [Ne III] & 0.21 & $9.7 \pm 3.5$ & 3.54: & $\ldots$ & $\ldots$ & $11.0 \pm 3.6$ & $13.2 \pm 3.7$ \\
\hline 4068 [S II] & 0.19 & 2.23: & $\ldots$ & $\ldots$ & $\ldots$ & $\ldots$ & $\ldots$ \\
\hline 4076 [S II] & 0.19 & 1.13: & $\ldots$ & $\ldots$ & $\ldots$ & $\ldots$ & $\ldots$ \\
\hline $4101 \mathrm{H} \delta$ & 0.18 & $25.9 \pm 4.3$ & $25.9 \pm 4.6$ & $25.9 \pm 4.8$ & $25.9 \pm 7.7$ & $25.9 \pm 4.4$ & $25.9 \pm 4.7$ \\
\hline $4340 \mathrm{H} \gamma$ & 0.135 & $46.9 \pm 4.7$ & $46.9 \pm 5.2$ & $46.9 \pm 7.2$ & $46.9 \pm 8.8$ & $46.9 \pm 8.1$ & $46.9 \pm 9.8$ \\
\hline 4363 [O III] & 0.13 & 1.76: & $\ldots$ & $\ldots$ & $\ldots$ & $\ldots$ & 1.85: \\
\hline $4471 \mathrm{He} \mathrm{I}$ & 0.10 & 2.14: & $3.90 \pm 1.5$ & $\ldots$ & $\ldots$ & 3.23: & $\ldots$ \\
\hline $4861 \mathrm{H} \beta$ & 0.00 & $100 \pm 5$ & $100 \pm 6$ & $100 \pm 5$ & $100 \pm 7$ & $100 \pm 5$ & $100 \pm 6$ \\
\hline 4959 [O III] & -0.02 & $32.2 \pm 2.3$ & $33.9 \pm 2.7$ & $78.2 \pm 5.4$ & $124 \pm 7$ & $69.0 \pm 5.5$ & $57.7 \pm 6.1$ \\
\hline 5007 [O III] & -0.03 & $97.4 \pm 5.9$ & $109 \pm 7$ & $218 \pm 10$ & $375 \pm 19$ & $201 \pm 12$ & $161 \pm 10$ \\
\hline $5016 \mathrm{He} \mathrm{I}$ & -0.03 & 1.18: & $\ldots$ & $\ldots$ & $\ldots$ & $\ldots$ & $\ldots$ \\
\hline $5200[\mathrm{~N} \mathrm{I}]$ & -0.05 & $\ldots$ & $2.97 \pm 0.79$ & $\ldots$ & $\ldots$ & $\ldots$ & $\ldots$ \\
\hline $5518[\mathrm{Cl} \mathrm{III}]$ & -0.17 & $\ldots$ & 0.94: & $\ldots$ & $\ldots$ & $\ldots$ & $\ldots$ \\
\hline $5876 \mathrm{He} \mathrm{I}$ & -0.23 & $10.3 \pm 1.2$ & $11.1 \pm 1.9$ & $12.6 \pm 2.2$ & $14.5 \pm 2.4$ & $10.3 \pm 1.3$ & $12.6 \pm 1.9$ \\
\hline $6300[\mathrm{O} \mathrm{I}]$ & -0.30 & $6.73 \pm 0.60$ & $7.56 \pm 0.76$ & $19.1 \pm 3.0$ & $9.7 \pm 2.2$ & $4.55 \pm 0.79$ & $4.9 \pm 1.9$ \\
\hline $6364[\mathrm{O} \mathrm{I}]$ & -0.31 & 1.83: & 1.77: & $\ldots$ & $\ldots$ & 1.41: & $\ldots$ \\
\hline 6548 [N II] & -0.34 & $31.4 \pm 2.7$ & $31.5 \pm 3.3$ & $8.0 \pm 1.7$ & $9.4 \pm 3.3$ & $14.2 \pm 1.9$ & $14.9 \pm 2.2$ \\
\hline $6563 \mathrm{H} \alpha$ & -0.34 & $286 \pm 11$ & $286 \pm 12$ & $286 \pm 12$ & $286 \pm 14$ & $286 \pm 12$ & $286 \pm 14$ \\
\hline 6584 [N II] & -0.34 & $102.2 \pm 6.4$ & $98.1 \pm 8.8$ & $22.6 \pm 2.7$ & $22.8 \pm 4.1$ & $43.1 \pm 3.0$ & $46.5 \pm 5.4$ \\
\hline $6678 \mathrm{He} \mathrm{I}$ & -0.35 & 1.84: & $\ldots$ & $\ldots$ & $10.3 \pm 2.9$ & 2.09: & $\ldots$ \\
\hline 6716 [S II] & -0.36 & $42.9 \pm 3.8$ & $43.8 \pm 3.9$ & $48.7 \pm 4.9$ & $34.8 \pm 4.3$ & $31.0 \pm 3.4$ & $32.0 \pm 5.7$ \\
\hline $6731[\mathrm{~S}$ II] & -0.36 & $36.0 \pm 2.9$ & $35.1 \pm 4.2$ & $37.4 \pm 4.1$ & $27.4 \pm 3.6$ & $23.7 \pm 2.2$ & $26.4 \pm 2.9$ \\
\hline
\end{tabular}

${ }^{a}[\mathrm{O}$ II] $\lambda \lambda 3726,3729$ emission flux.

have assumed a two-zone scheme, the usual relation $\mathrm{O} / \mathrm{H}=$ $\mathrm{O}^{+} / \mathrm{H}^{+}+\mathrm{O}^{++} / \mathrm{H}^{+}$and the linear relation between $T([\mathrm{O} \mathrm{III}])$ and $T([\mathrm{OII}])$ based on photoionization models obtained by Garnett (1992):

$T([\mathrm{O} \mathrm{II}])=0.7 \cdot T([\mathrm{O} \mathrm{III}])+3000$.

The empirical relation between the oxygen abundance and the $T([\mathrm{O}$ III]) given by Pilyugin (2001) (his Eq. (11)) gives similar $T[\mathrm{O}$ III] results than the ones obtained before. The finally adopted $T[\mathrm{O}$ III $]$ and $T[\mathrm{O}$ II $]$ for each burst are shown in Table 3. Following Pilyugin (2001), we could assume an uncertainty between 500 and $1000 \mathrm{~K}$ for $T$ [O III].

We have used the [S II] $\lambda \lambda 6717,6731$ doublet to determine the electron density, $N_{\mathrm{e}}$, of the ionized gas. Electron densities were calculated making use of the five-level program for the analysis of emission-line nebulae that is included in IRAF NEBULAR task (Shaw \& Dufour 1995). For zones \#14 and \#15, electron densities are below the low-density limit $\left(<100 \mathrm{~cm}^{-3}\right)$. We have no determination of $N_{\mathrm{e}}$ for \#1 and \#3. The final adopted values of $T_{\mathrm{e}}$ and $N_{\mathrm{e}}$ (or their upper limits) are compiled in Table 3.

\subsubsection{Abundance analysis}

We have derived the total oxygen abundance making use of empirical calibrations, based on the relative intensities of strong optical lines. Denicoló et al. (2002) used the [N II] $\lambda 6583 / \mathrm{H} \alpha$ ratio in combination with photoionization models to obtain the calibration:

$12+\log \frac{\mathrm{O}}{\mathrm{H}}=9.2+0.73 \log \frac{[\mathrm{N} \mathrm{II}] 6583}{\mathrm{H} \alpha}$.

However, the most widely used empirical abundance calibrator is the $R_{23}$ parameter, defined as (Pagel et al. 1980):

$R_{23} \equiv \frac{I([\mathrm{O} \mathrm{II}] 3727)+I([\mathrm{O} \mathrm{III}] 4959+5007)}{I(\mathrm{H} \beta)}$.

Pilyugin (2000) found that the previous calibrations using this parameter had a systematic error depending on the hardness of the ionizing radiation, suggesting that the excitation parameter, $P$, is a good indicator of it. This parameter was defined as:

$P \equiv \frac{I([\mathrm{O} I \mathrm{II}] 4959+5007)}{I(\mathrm{H} \beta)} \frac{1}{R_{23}}$ 
Table 5. Dereddened line intensity ratios with respect to $I(\mathrm{H} \beta)=100$ for the faint knots observed in Mkn 1087. Correction for underlying stellar absorption in H I Balmer lines was also applied. All spectra were taken using INT.

\begin{tabular}{lccccccc}
\hline \hline Line & $f(\lambda)$ & $\# 1$ & $\# 3$ & $\# 8$ & $\# 13$ & $\# 14$ & $\# 15$ \\
\hline $3727[\mathrm{O} \mathrm{II}]$ & 0.27 & $265:$ & $240:$ & $290 \pm 35$ & $252 \pm 75$ & $271 \pm 26$ & $286.39:$ \\
$3889 \mathrm{He} \mathrm{I}+\mathrm{H} 8$ & 0.22 & $\ldots$ & $\ldots$ & $19.5 \pm 6.4$ & $\ldots$ & $\ldots$ & $\ldots$ \\
$4340 \mathrm{H} \gamma$ & 0.135 & $\ldots$ & $\ldots$ & $46.9 \pm 7.9$ & $\ldots$ & $47 \pm 14$ & $\ldots$ \\
$4861 \mathrm{H} \beta$ & 0.00 & $100:$ & $100:$ & $100 \pm 9$ & $100 \pm 23$ & $100 \pm 12$ & $100:$ \\
$4959[\mathrm{O} \mathrm{III}]$ & -0.02 & $\ldots$ & $\ldots$ & $56.7 \pm 8.5$ & $55 \pm 14$ & $50.7 \pm 6.6$ & $34.7:$ \\
$5007[\mathrm{O} \mathrm{III}]$ & -0.03 & $136:$ & $99:$ & $165 \pm 14$ & $144 \pm 22$ & $173 \pm 14$ & $86.5:$ \\
$5876 \mathrm{HeI}$ & -0.23 & $\ldots$ & $\ldots$ & $\ldots$ & $\ldots$ & $11.4 \pm 4.1$ & $\ldots$ \\
$6548[\mathrm{~N} \mathrm{II}]$ & -0.34 & $\ldots$ & $\ldots$ & $16.8 \pm 5.9$ & $26 \pm 10$ & $13.0 \pm 4.5$ & $\ldots$ \\
$6563 \mathrm{H} \alpha$ & -0.34 & $286 \pm 46$ & $286 \pm 54$ & $286 \pm 23$ & $286 \pm 31$ & $286 \pm 10$ & $286 \pm 34$ \\
$6584[\mathrm{~N} \mathrm{II}]$ & -0.34 & $54 \pm 18$ & $51.5:$ & $60.0 \pm 9.1$ & $51.0 \pm 7.1$ & $59.7 \pm 7.2$ & $71 \pm 25$ \\
$6716[\mathrm{~S} \mathrm{II}]$ & -0.36 & $\ldots$ & $\ldots$ & $45.7 \pm 7.4$ & $46.7 \pm 7.6$ & $43.2 \pm 6.5$ & $22:$ \\
$6731[\mathrm{~S} \mathrm{II}]$ & -0.36 & $\ldots$ & $\ldots$ & $36.0 \pm 7.9$ & $36.0 \pm 5.8$ & $27.4 \pm 5.4$ & $57:$ \\
\hline
\end{tabular}

Table 6. Chemical abundances of the knots.

\begin{tabular}{|c|c|c|c|c|c|c|c|c|c|c|c|c|}
\hline Knot & C WHT & C INT & N WHT & N INT & \#7 WHT & \#7 INT & $\# 1$ & \#3 & $\# 8$ & $\# 13$ & $\# 14$ & $\# 15$ \\
\hline $12+\log \mathrm{O} / \mathrm{H}^{a}$ & 8.57 & 8.53 & 8.23 & 8.24 & 8.55 & 8.53 & 8.50: & 8.52: & 8.41 & 8.47 & 8.43 & 8.44 \\
\hline $12+\log \mathrm{O} / \mathrm{H}^{b}$ & 8.79 & 8.78 & 8.31 & 8.32 & 8.52 & 8.54 & 8.59 & 8.58 & 8.63 & 8.57 & 8.62 & 8.68 \\
\hline$\left(\mathrm{O}^{++}+\mathrm{O}^{+}\right) / \mathrm{O}^{+}$ & 1.55 & 1.53 & 1.53 & 1.99 & 2.19 & 1.85 & $\ldots$ & $\ldots$ & 1.57 & 1.64 & 1.61 & 1.67 \\
\hline $12+\log \mathrm{N} / \mathrm{H}$ & 7.76 & 7.71 & 6.77 & 6.90 & 7.48 & 7.43 & $\ldots$ & $\ldots$ & 7.33 & 7.48 & 7.32 & 7.32 \\
\hline$-\log \mathrm{N}^{+} / \mathrm{O}^{+}$ & 0.81 & 0.83 & 1.46 & 1.34 & 1.08 & 1.10 & $\ldots$ & $\ldots$ & 1.08 & 0.99 & 1.11 & 1.00 \\
\hline $12+\log \mathrm{Ne}^{++} / \mathrm{H}^{+}$ & 7.67 & 7.60 & 7.53 & 7.42 & 7.56 & 7.79 & $\ldots$ & $\ldots$ & $\ldots$ & $\ldots$ & $\ldots$ & $\ldots$ \\
\hline $12+\log \mathrm{S}^{+} / \mathrm{H}^{+}$ & 6.54 & 6.50 & 6.24 & 6.07 & 6.31 & 6.34 & $\ldots$ & $\ldots$ & 6.38 & 6.44 & 6.33 & 6.45 \\
\hline $12+\log \mathrm{S}^{++} / \mathrm{H}^{+}$ & 6.42 & $\ldots$ & $\ldots$ & $\ldots$ & $\ldots$ & $\ldots$ & $\ldots$ & $\ldots$ & $\ldots$ & $\ldots$ & $\ldots$ & $\ldots$ \\
\hline $12+\log \mathrm{He}^{+} / \mathrm{H}^{+c}$ & 10.84 & 10.88 & 10.97 & 11.03 & 10.82 & 10.94 & $\ldots$ & $\ldots$ & $\ldots$ & $\ldots$ & 10.91 & $\ldots$ \\
\hline$[\mathrm{O} / \mathrm{H}] /[\mathrm{O} / \mathrm{H}]_{\odot}{ }^{d}$ & 0.76 & 0.69 & 0.35 & 0.35 & 0.72 & 0.69 & $0.72:$ & $0.65:$ & 0.60 & 0.71 & 0.55 & 0.56 \\
\hline
\end{tabular}

${ }^{a}$ Determined using the empirical calibration of Pilyugin (2001).

${ }^{b}$ Determined using the $[\mathrm{N} \mathrm{II}] / \mathrm{H} \alpha$ ratio (Denicoló et al. 2002).

${ }^{c}$ Using only the $\mathrm{He} \mathrm{I} \lambda 5875$ emission line.

${ }^{d}$ Asuming $12+\log [\mathrm{O} / \mathrm{H}]_{\odot}=8.69 \pm 0.05$ (Allende Prieto et al. 2001).

Pilyugin $(2000,2001)$ performed a detailed analysis of the observational data combined with photoionization models to obtain empirical calibrations for the oxygen abundance. Pilyugin (2000) proposes a linear fit that involves only the $R_{23}$ parameter (for $12+\log (\mathrm{O} / \mathrm{H})>8.15$ ), whereas Pilyugin (2001) is the following, more real and complex, calibration involving also the excitation parameter:

$$
12+\log \frac{\mathrm{O}}{\mathrm{H}}=\frac{R_{23}+54.2+59.45 P+7.31 P^{2}}{6.01+6.71 P+0.371 P^{2}+0.243 R_{23}},
$$

(the so-called $P$-method), that can be used in moderately high-metallicity $\mathrm{H}$ II regions.

In Table 6 we show the oxygen abundances obtained for each observed knot using both empirical calibrations. We have assumed an error of $\sim \pm 0.2$ dex for the oxygen abundances using Denicoló et al. (2002). Pilyugin (2001) estimates an uncertainty of \pm 0.10 dex for his calibration. We find that the $\mathrm{O} / \mathrm{H}$ ratios obtained using the calibration of Denicoló et al. (2002) are systematically slightly higher that those obtained using the calibrations of Pilyugin (2001). Only in the bright burst \#7 both calibrations give a similar abundance. Denicoló et al. (2002) remarked that their calibration has a scatter that seems to depend on the degree of ionization. In consequence, we have adopted the oxygen abundances derived using the P-method as the more appropiates ones.

Note that the north companion object, N, has a clearly lower oxygen abundance [around 8.24, in units of $12+\log (\mathrm{O} / \mathrm{H})$ ] compared with the rest of the knots (between 8.41 and 8.57). Vacca \& Conti (1992) reported an oxygen abundance of 8.55 for the main body of Mkn 1087 assuming $T_{\mathrm{e}}=10000 \mathrm{~K}$ and $N_{\mathrm{e}}=340 \mathrm{~cm}^{-3}$, value that coincides with the abundance we obtain for the central zone of the galaxy. The average value among all the observed knots inside Mkn 1087 is $12+\log (\mathrm{O} / \mathrm{H})=8.49$.

In Table 6 we list the ionic and $\mathrm{O}$ abundances we have derived. All the ionic abundances have been calculated using the IRAF NEBULAR task from the intensity of collisionally excited lines, assuming the $T([\mathrm{O} \mathrm{III}]$ ) for the high ionization potential ions $\mathrm{O}^{++}, \mathrm{Ne}^{++}$, and $\mathrm{S}^{++}$, and $T([\mathrm{OII}])$ for the low ionization potential ions $\mathrm{O}^{+}, \mathrm{N}^{+}$and $\mathrm{S}^{+}$. 

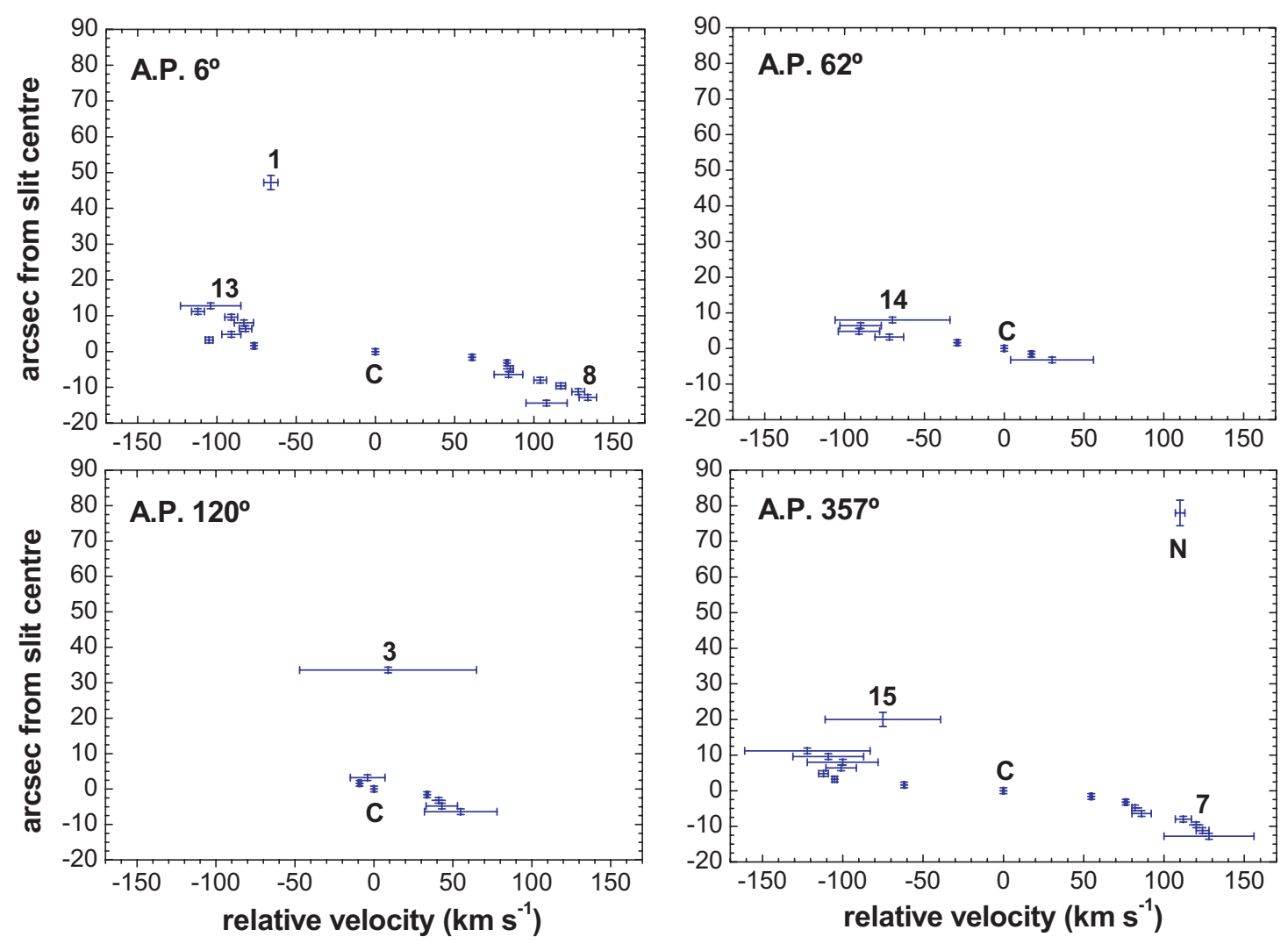

Fig. 5. a), b), c) and d): position-velocity diagrams for the four slit positions observed with INT, analysed in 1.6 arcsec bins. The horizontal bars represent the uncertainty of the Gaussian fitting for each point. The location and extension of the different galaxy members are also indicated. North is up in all the diagrams.

We have assumed the standard ionization correction factor (icf) by Peimbert \& Costero (1969), $\mathrm{N} / \mathrm{O}=\mathrm{N}^{+} / \mathrm{O}^{+}$, to derive the nitrogen abundance of the objects. This correction factor is listed in Table 6. Except for the central zone of Mkn 1087, only the $\mathrm{S}^{+} / \mathrm{H}^{+}$ratio is derived, so we are not able to derive the total sulphur abundance. Furthermore, some contribution of $\mathrm{S}^{3+}$ is also expected in the areas with a higher ionization degree.

The $\mathrm{He}^{+} / \mathrm{H}^{+}$ratio has been derived from the $\mathrm{He}$ I $\lambda 5875$ line observed in each knot and using the predicted line emissivities calculated by Smith et al. (1996). We have also corrected for collisional contribution following the calculations by Benjamin et al. (2002). In Table 6 we present the $\mathrm{He}^{+} / \mathrm{H}^{+}$ratios finally obtained. The results obtained from INT and WHT data for a given object are quite similar, despite the uncertainties. This indicates the good quality of the spectra and the reduction and analysis procedures.

\subsubsection{Kinematics of Mkn 1087}

The kinematics of the ionized gas were studied via the spatially resolved analysis of bright emission line profiles $(\mathrm{H} \alpha$ and $\mathrm{H} \beta$ for INT and WHT data, respectively) along each slit position. We have extracted zones of 4 pixels wide (1.6" long) covering all the extension of the line-emission zones in the four INT slit positions shown in Fig. 3, and zones of 6 pixels wide (1.2" long) for the WHT slit position. The Starlink DIPSO software was used to perform a Gaussian fitting to the $\mathrm{H} \alpha$ and $\mathrm{H} \beta$ profiles. In Figs. 5 and 6 we show the position-velocity diagrams for the four INT slit positions and the single WHT slit position, respectively. All the velocities are referred to the mean heliocentric velocity of the center of Mkn $1087\left(8336 \mathrm{~km} \mathrm{~s}^{-1}\right)$. The position of the different objects is also indicated in Figs. 5 and 6 . In Table 3 we show the mean radial velocities obtained for each zone, that span a narrow interval of values with a maximum difference of about $250 \mathrm{~km} \mathrm{~s}^{-1}$.

All the position-velocity diagrams show a more or less clear rotation pattern in the inner part of Mkn 1087. Knot \#7 participates of this rotation. However, the external zones have significant deviations from this behavior. Knots \#1 and \#3 are kinematically attached to the main body of the system despite their separation. We suggest that they are tidal dwarf galaxies (TDGs) formed from material stripped from Mkn 1087. Objects \#13, \#14 and \#15 seem to be kinematically attached to the rotation of Mkn 1087. There is a small slightly sinusoidal pattern in the gas velocity around \#13. This behavior, if real, could be produced by distortions associated with interaction effects (Schweizer 1982; Rubin et al. 1990). Note that knot \#1 has a velocity similar to \#13. The image of the system (see Fig. 1) reveals an optical bridge between both objects, that furthermore have a very similar metallicity $[12+\log (\mathrm{O} / \mathrm{H}) \sim$ 8.50]. Figure 6 also shows some areas that can be interpreted as a tidal tail to knot \#1. All these evidences reinforce the idea that this structure (that has \#1 and \#13 at its extremes) could be actually a tidal tail. 


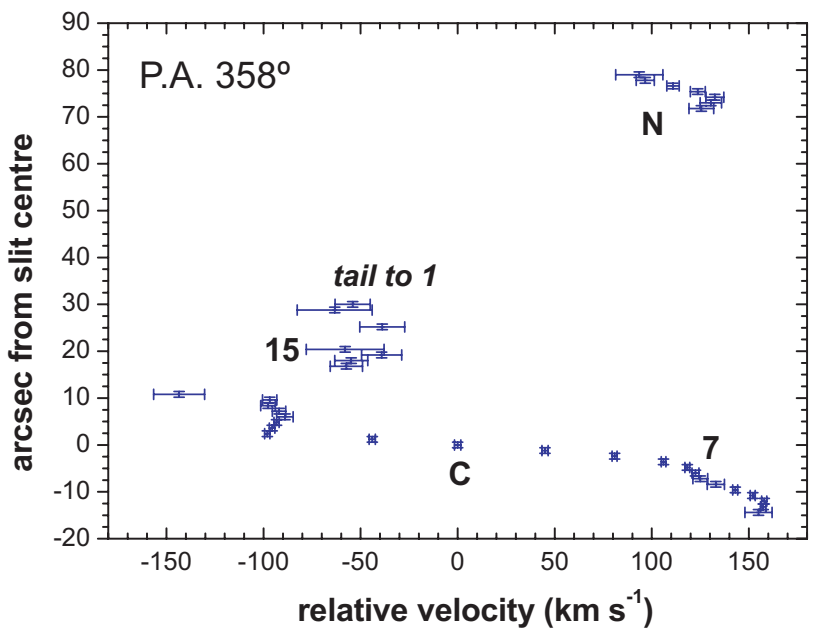

Fig. 6. Position-velocity diagram for the single slit position observed in Mkn 1087 with WHT, analysed in 1.2 arcsec bins. The horizontal bars represent the uncertainty of the Gaussian fitting for each point. The location and extension of the different galaxy members are also indicated. North is up.

The HST image of the center of Mkn 1087 (Malkan et al. 1998) shows a clear spiral pattern, implying a dynamically mature system. It also reveals that its west side is nearer to us. From the position-velocity diagrams of PA $357^{\circ}$ and $358^{\circ}$, it is clear that the north side of the spiral pattern is the approaching one. If these two diagrams are interpreted as a circular rotation, then we can estimate the Keplerian mass of Mkn 1087. Taking into account the half of the maximum velocity difference $\left(\sim 125 \mathrm{~km} \mathrm{~s}^{-1}\right)$ and the half of the spatial separation corresponding to these maxima $\left(\sim 12^{\prime \prime} \simeq 6500 \mathrm{pc}\right)$, we obtain a mass of $2.37 \times 10^{10} M_{\odot}$ for an inclination of $90^{\circ}$, assuming circular orbits and Keplerian dynamics:

$\frac{M}{M_{\odot}} \sim 233 \times d[\mathrm{pc}] \cdot\left(\frac{v\left[\mathrm{~km} \mathrm{~s}^{-1}\right]}{\sin i}\right)^{2}$.

A similar result is found using the position-velocity diagram of PA $6^{\circ}$. If we use PA $62^{\circ}$ we estimate a velocity difference of around $100 \mathrm{~km} \mathrm{~s}^{-1}$ in $8^{\prime \prime}(\simeq 4300 \mathrm{pc})$, obtaining a Keplerian mass of $1.0 \times 10^{10} M_{\odot}$, assuming an inclination of $90^{\circ}$. If we consider that the elliptical shape of Mkn 1087 is only due to its inclination with respect the line of sight, we find that the proportion between the mayor axis (that correspond with PA $357^{\circ}$ ) and the minor axis (between PA $62^{\circ}$ and $120^{\circ}$ ) of the assumed disk is $5 / 3$. This value corresponds with an inclination (defined as the angle between the plane of the sky and the plane of the galaxy) of $37^{\circ}$ [Gordon \& Gottesman (1981) derived an inclination angle of $38^{\circ}$. We now obtain a Keplerian mass of $6.53 \times 10^{10} M_{\odot}$ for PA $357^{\circ}$ (mayor axis) and $4.63 \times$ $10^{10} M_{\odot}$ for PA $62^{\circ}$ (minor axis). For the last one, we have also corrected the projected distance, and it is the $70 \%$ of the mass obtained from the position-velocity diagram of PA $357^{\circ}$. The average value between both estimations gives a Keplerian mass of $M_{\mathrm{kep}}=5.6 \times 10^{10} M_{\odot}$ for Mkn 1087. This value corresponds to a light to Keplerian mass ratio of $L_{B} / M_{\mathrm{kep}} \sim 2.19$. Gordon \& Gottesman (1981), using the Arecibo antenna, estimated a total hydrogen mass of $M_{\mathrm{HI}}=1.72 \times 10^{10} M_{\odot}$ and a total dynamical mass of $M_{\text {dyn }}=1.78 \times 10^{11} M_{\odot}$ [from the velocity width of the H I profile and applying the Brandt (1960) rotation law], that give a light to $\mathrm{HI}$ mass ratio of $L_{B} / M_{\mathrm{HI}} \sim 7.12$ and a light to dynamical mass ratio of $L_{B} / M_{\mathrm{dyn}} \sim 0.69$. Our $L_{B} / M_{\text {kep }}$ ratio agrees with these estimates. Typical $L_{B} / M_{\mathrm{HI}}$ values for spiral galaxies are between 11.2 and 8.9 (Bettoni et al. 2003). Consequently, Mkn 1087 is a relatively gas-rich system.

Keel (1988) obtained position-velocity diagrams using various emission lines $(\mathrm{H} \alpha, \mathrm{H} \beta,[\mathrm{NII}],[\mathrm{O} I I I])$, instruments and detectors for PA $57^{\circ}$ (this slit position crosses knot \#2) and for PA $68^{\circ}$. The last one is very similar to the position-velocity diagram we obtain for PA $62^{\circ}$. The outer parts of the emission detected by Keel (1988) (that was chosen because it corresponds to one of the observed filaments) are redshifted by $\sim 200 \mathrm{~km} \mathrm{~s}^{-1}$ with respect the inner part of Mkn 1087. He suggested that this feature indicates that this filament is infalling. However, as we will comment in Sect. 4.6, we think that the observed filaments in Mkn 1087 are tidal tails originated due to interaction with the nearby galaxy KPG 103a and the north companion object.

The companion object has a radial velocity similar to the main body of Mkn $1087\left(+117 \mathrm{~km} \mathrm{~s}^{-1}\right)$, but it is kinematically detached of the rotation pattern of the main galaxy. In Fig. 6 we can observe that the north companion object seems to have a sort of small rotation pattern, although its southern tip shows if real - a deviation that could be interpreted as a feature of the interaction between it and Mkn 1087. Assuming Keplerian rotation and an inclination of $90^{\circ}$, we estimated a mass of $2.2 \times$ $10^{8} M_{\odot}$ for this object, just two orders of magnitude lower than the value estimated for Mkn 1087. Typical values of the mass for dwarf galaxies are between $10^{7}$ and $10^{8} M_{\odot}$, so we can classify it as a dwarf object. These kinematic results, together with its low oxygen abundance $(\sim 8.24)$ reinforce the evidence that it is an external galaxy and not a TDG.

We remark the fact that knot \#7 shows no important deviation of the main rotation pattern of Mkn 1087 in diagrams with PA $357^{\circ}$ and $358^{\circ}$. This fact does not support the Keel (1988) suggestion that \#7 is consequence of the merger of a dwarf galaxy. In the best case, this presumed merger should be in a very advanced stage. Our data is more consistent with the interpretation of knot \#7 as a giant $\mathrm{H}$ II region (or a complex of bright star-forming regions) located in one of the spiral arms of Mkn 1087.

\section{Discussion}

\subsection{Ages of the bursts and stellar populations}

ME00 used Leitherer \& Heckman (1995) models to derive the ages of the bursts using their optical photometric data. We have improved their results combining our reddening-corrected broad-band optical and near-infrared photometric data with STARBURST99 (Leitherer et al. 1999) models (the improvement of Leitherer \& Heckman models). Spectral synthesis models with two different metallicities were chosen $\left(Z / Z_{\odot}=\right.$ 1 and 0.4 , the appropriate range of metallicities for the objects, see Table 6), both assuming an instantaneous burst with 

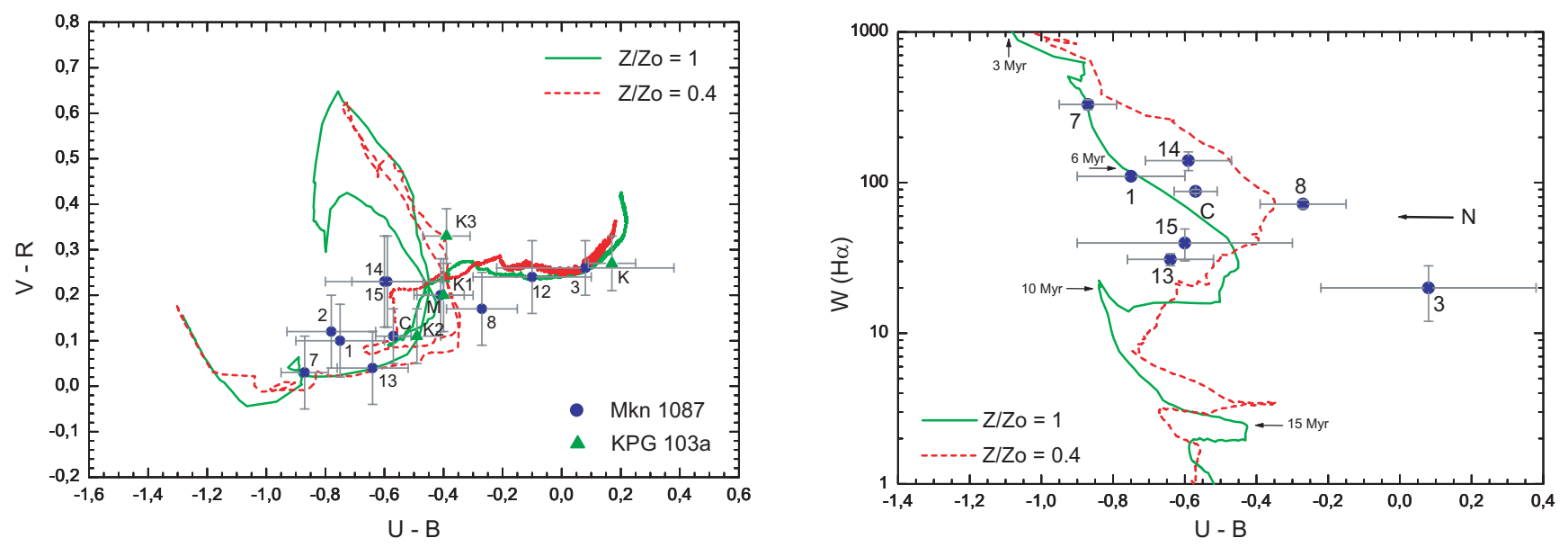

Fig. 7. a) $(V-R)$ versus $(U-B)$ values of the knots and STARBURST99 (Leitherer et al. 1999) predictions for an instantaneous burst with a Salpeter IMF and $Z / Z_{\odot}=1$ and 0.4. The age of the bursts decrease from left to right. M represents the values for Mkn 1087 main body, whereas $\mathrm{K}$ represents the values for the galaxy KPG 103a. b) $W(\mathrm{H} \alpha)$ versus $(U-B)$ for knots in Mkn 1087 and STARBURST99 (Leitherer et al. 1999) predictions. Some ages of the $Z / Z_{\odot}=1$ model are marked, showing the temporal evolution of the burst. The $W(\mathrm{H} \alpha)$ of the north companion object is indicated with an horizontal arrow because there is not $U-B$ color available for it.

a Salpeter IMF, a total mass of $10^{6} M_{\odot}$ and a $100 M_{\odot}$ upper stellar mass. The different color-color diagrams gave a similar interval of ages for all objects. In Fig. 7a the predictions of $(V-R)$ versus $(U-B)$ are compared with our observational values. Knots with redder colors $(\# 12, \# 3$ and the bright galaxy KPG 103a) correspond to the older objects in the system (knot \#11 should be included in this "red-group"). We have estimated an age of around $150 \mathrm{Myr}$ for these three knots, values similar to those obtained by ME00.

A detailed analysis of the procedure used to derive the age is described in our study of the Hickson Compact Group 31 (López-Sánchez et al. 2004). We have also used the Wolf-Rayet starburst models by Schaerer \& Vacca (1998) and Stasińska et al. (2001) models to derive the age of the bursts using spectral features. A good agreement in all the age estimations has been obtained. We show the finally adopted value of the age of each spectroscopically studied burst in Table 3 . We have found that all the starbursts are between 6 and 9 Myr old, except knot \#7 that seems to be younger (4.5 Myr).

In Fig. 7b we plot our observational values of $\mathrm{W}(\mathrm{H} \alpha)$ (obtained from the spectroscopic data) versus the color $(U-B)$ (derived from the photometric data), and the predictions of the STARBURST99 models. We find again a good agreement with the models except for knot \#3, an older object that hosts a little star-formation activity.

As we commented before, the three bright knots ( $\mathrm{K} 1$, K2 and K3) inside KPG 103a show blue colors (see their $(U-B)$ colors in Table 2). Although we have no spectra for them, they seem to be starburst zones in the nucleus of the galaxy. We have used the same STARBURST99 (Leitherer et al. 1999) models to estimate the age of the star-formation in them. As it can be seen in Fig. 7a, the position of K1, K2 and $\mathrm{K} 3$ are consistent with the models, and we estimate an age between 6 and $7 \mathrm{Myr}$ for them. However, if the color excess, $E(B-V)$, were higher than the one we have addopted (we only considered the Galactic contribution to the reddening for them,

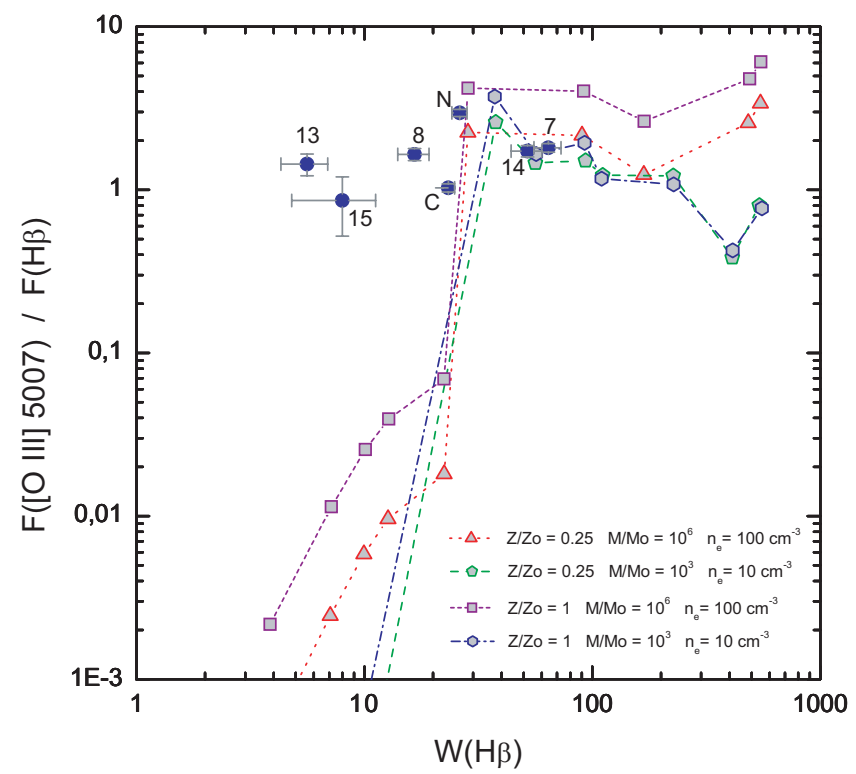

Fig. 8. $\mathrm{F}([\mathrm{O} \mathrm{III}]$ 15007) versus $\mathrm{W}(\mathrm{H} \beta)$. Models by Stasińska et al. (2001). Tracks correspond to sequences of different metallicities and electronic densities. Each symbol marks the position of the models at $1 \mathrm{Myr}$ interval, starting in the upper-right corner of the diagram with an age of $1 \mathrm{Myr}$.

see Sect. 3.1), these knots will show bluer colors and, consequently, they will be a slightly younger.

In Fig. 8 we plot our observational values of $W(\mathrm{H} \beta)$ and the [O III] $\lambda 5007 / \mathrm{H} \beta$ emission line flux showed in Tables 3 and 4 , respectively, compared with the Stasińska et al. (2001) models of $\mathrm{H}$ II regions ionized by an evolving starburst embedded in a gas cloud of the same metallicity. We indicate four models with two metallicities, $Z / Z_{\odot}=0.25$ and $Z / Z_{\odot}=1$, changing the total mass and electronic density of the ionized gas. All the objects show a good fit with the predictions, except knots \#13 and \#15. This fact can be due to a substantial population of old 
stars present in those objects, although it is difficult to detect them in the spectra. However, as we saw before, their colors and $W(\mathrm{H} \alpha)$ indicate that they host a starburst that are around 8 Myr old.

The spectrum of the center of Mrk 1087 (see Fig. 4a) shows clear Hi Balmer line absorptions, so we can estimate an effective age for the stellar population in the nucleus using the STARBURST99 (Leitherer et al. 1999) models. The best fit gives an age around $100 \mathrm{Myr}$, suggesting that star formation has been active in the nucleus of the galaxy ever since.

In order to investigate the importance of older stellar populations, we have performed a simple analysis of the surface brightness profiles of the two main galaxies, as well as the north companion object and knot \#3. We have taken concentric surfaces at different radii from the center of each system, and calculated the integrated flux inside each circle of area $A$ using the relation:

$\mu_{X}=m_{X}+2.5 \log A$,

to obtain the surface brightness, $\mu$ (in units of mag $\operatorname{arcsec}^{-2}$ ), of each circle $A$ (in units of $\operatorname{arcsec}^{2}$ ) and $m_{X}$ is the magnitude in the filter $X$. In Fig. 9 we show the surface brightness for $B$, $V$ and $R$ filters versus the size of the aperture (in arcsec) for Mkn 1087 and KPG 103a, whereas the surface brightness for the north companion and object \#3 are shown in Fig. 10. In these two figures we also show the radial color profiles $(B-R)$ and $(B-V)$ (this last only in Fig. 9) derived by a direct subtraction of light profiles. The dotted horizontal line indicates the representative average color derived for each system (see Table 2).

We can observe that the surface brightness profile can be separated in two structures in all objects except \#3, especially in the case of the $B$-band, indicating that they have a low surface brightness component underlying the starburst. In the case of the two brightest galaxies, we can attribute this underlying component to the galactic disk. We have performed an exponential law fitting to the profiles, following the expression:

$I=I_{0} \exp (-\alpha r)$

that describes a typical disk structure: $I_{0}$ is the central intensity and $\alpha$ is the scale length. The fitting structural parameters are indicated in Table 7 and the fit is plotted over each $B$ profile with a straight line. It is not a surprise to find two structural components in Mkn 1087 and KPG 103a: Fig. 2 indicates the presence of a galactic disk with a possible spiral structure in both galaxies. Furthermore, it is interesting to remark the noticeable variation in the $(B-V)$ color along KPG 103a: it seems that the star-formation activity is very concentrated at its center, probably in the three inner knots (K1, K2 and K3) analyzed in Sect. 3.1. Note that they show considerably bluer colors, $(U-B) \sim-0.4$, than the one derived for the complete galaxy, $(U-B)=0.17$. We also find two structures in the north companion object (Fig. 10), indicating that an important old population is present and further supporting the idea that this object actually is an external galaxy nad not a tidal dwarf. However, we do not find two components in knot \#3. Is the contribution of the starburst dominating the underlying population? This problem
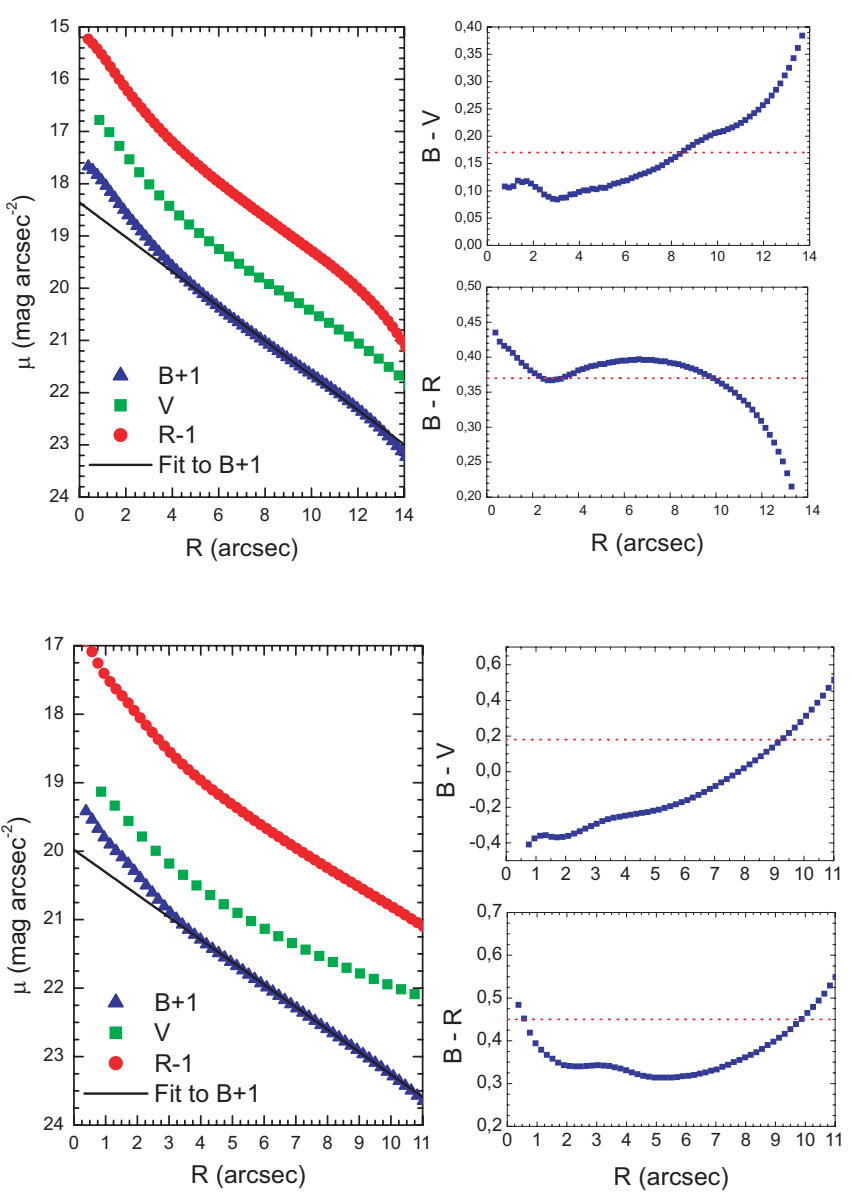

Fig. 9. Surface brightness and $B, V$ and $R$ color profiles for Mkn 1087 (up) and KPG 103a (down). The line in the surface brightness diagrams is an exponential law fitting to the $B$ profile. The dotted horizontal line in the color profile diagrams indicates the average color derived for each system.

of the "missing populations" in starbursts can be solved using high spatial resolution photometry in NIR (Noeske et al. 2003). However, we do not think that it could be applied in \#3: its low star-formation activity, colors and age suggest that most of the structure observed corresponds to an old stellar population. The weak contribution of the starburst is perhaps, in this particular case, diluted under the dominant old component.

\subsection{Star formation rates and the classification of Mkn 1087}

Mkn 1087 and KPG 103a were detected jointly by IRAS (InfraRed Astronomical Satellite) at 25, 60 and $100 \mu \mathrm{m}$ (source F04470+0314), with fluxes of $f_{25}=0.50 \mathrm{Jy}, f_{60}=$ $3.48 \mathrm{Jy}$ and $f_{100}=4.77 \mathrm{Jy}$, respectively (IRAS Point Source Catalog, 1986). Using the Kennicutt (1998) calibration, we find that the star formation rate (SFR) derived for the system from infrared fluxes is $S F R_{\mathrm{IR}}=11.5 M_{\odot} \mathrm{yr}^{-1}$. It is a typical value for star formation galaxies. We can also estimate the dust temperature, $T_{\mathrm{D}}$, and the warm dust mass, $M_{\mathrm{D}}$, using the expressions given by Bettoni et al. (2003) and the $f_{60}$ and $f_{100}$ fluxes. We obtain $T_{\mathrm{D}}=43.2 \mathrm{~K}$ and $M_{\mathrm{D}}=7.62 \times 10^{6} M_{\odot}$. Considering the 
Table 7. Structural parameters of Mkn 1087 and the brightest galaxies and objects associated with it.

\begin{tabular}{cccccccc}
\hline \hline Member & U.C. $^{a}$ & $\mu_{B, 0}$ & $\alpha_{B}(\mathrm{kpc})$ & $\mu_{V, 0}$ & $\alpha_{V}(\mathrm{kpc})$ & $\mu_{R, 0}$ & $\alpha_{R}(\mathrm{kpc})$ \\
\hline Mkn 1087 & yes & 17.36 & 1.63 & 17.34 & 1.72 & 16.85 & 1.55 \\
KPG 103a & yes & 18.98 & 1.64 & 19.78 & 2.44 & 18.77 & 1.76 \\
N. comp. & yes & 20.91 & 1.22 & $\ldots$ & $\ldots$ & 20.89 & 1.22 \\
$\# 3$ & no & 21.93 & 1.42 & $\ldots$ & $\ldots$ & 21.52 & 1.41 \\
\hline
\end{tabular}

${ }^{a}$ Underlying component.
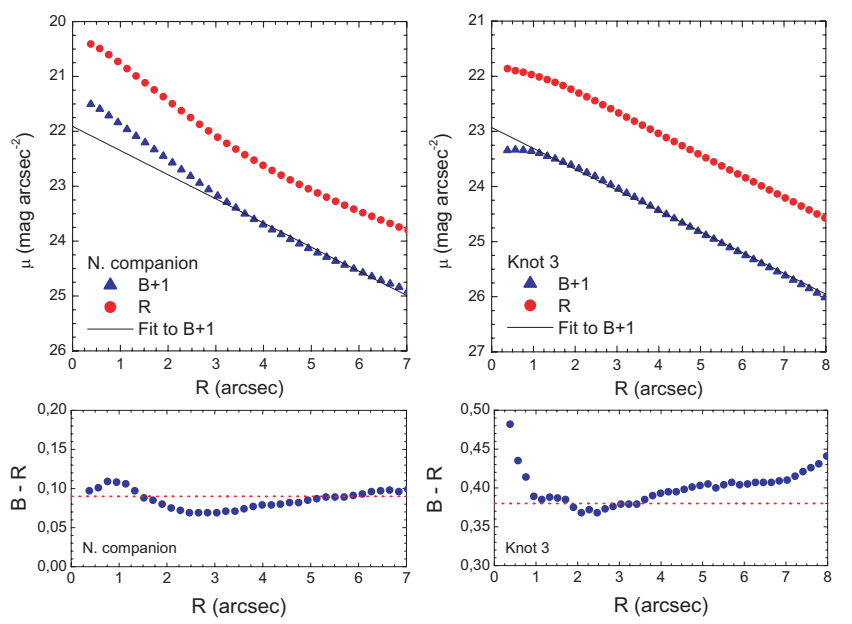

Fig. 10. Surface brightness and color profiles for the north companion object and knot \#3. We follow the same notation than in Fig. 9. Note that knot \#3 has not an underlying component.

luminosity of both galaxies, it corresponds to a mass to light ratio of $\log M_{\mathrm{D}} / L_{B}=-4.36$, a little lower than the expected values for spirals (between -3.74 and -4.07 , Bettoni et al. 2003).

Radio emission from galaxies is an extinction-free tracer of star formation. Condon et al. (2002) derived $\log L_{1.4 \mathrm{GHz}}=$ 22.33 for Mkn 1087 using the NRAO VLA Sky Survey (NVSS). We can estimate the SFR from the $1.4 \mathrm{GHz}$ luminosity using this data and the calibration given by Condon et al. (2002). We obtain a value of $S F R_{1.4 \mathrm{GHz}}\left(M>5 M_{\odot}\right) \sim$ $4.65 M_{\odot} \mathrm{yr}^{-1}$, that could be considered as the rate of massive star formation during the past $10^{8} \mathrm{yr}$. Assuming a Salpeter initial mass function between 0.1 and $100 M_{\odot}$, we can extrapolate it to include lower masses, finding $\operatorname{SFR}_{1.4 \mathrm{GHz}}\left(M>0.1 M_{\odot}\right) \sim$ $25.8 M_{\odot} \mathrm{yr}^{-1}$, a relative high value compared with the SFR derived from FIR fluxes. This is a reasonable result because $S F R_{1.4 \mathrm{GHz}}$ measures the SFR of the most recent bursts of the star formation, while $S_{F R}$ indicates the average SFR of a stellar population with larger average life times.

The SFR can be also derived using the $\mathrm{H} \alpha$ emission, applying the calibration given by Kennicutt (1998). Although we do not have $\mathrm{H} \alpha$ imagery of the system, we can make an estimation of the $\mathrm{H} \alpha$ from our spectra considering the slit size with respect to the total area of the bursts. We have estimated that the $\mathrm{H} \alpha$ flux (reddening corrected) we have measured in $\mathrm{C}$ is $\sim 10 \%$ of the total of the galaxy. This value corresponds to $S F R_{\mathrm{H} \alpha} \sim$ 4.5 $M_{\odot} \mathrm{yr}^{-1}$ for Mkn 1087. As we expect, this approximated value is lower than the ones derived from FIR and $1.4 \mathrm{GHz}$ fluxes because it does not take into account the dust-obscured
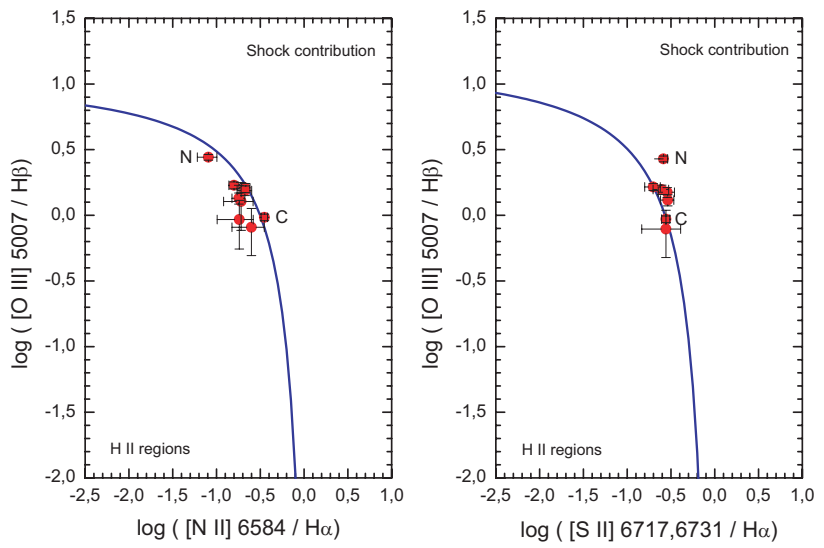

Fig. 11. Emission line ratios of knots in Mkn 1087. The solid lines give the limit for ionization by a zero age starburst, following Dopita et al. (2000).

areas. The probable range for the real SFR in Mkn 1087 is between 4.5 (from $\mathrm{H} \alpha$ flux) and 11.5 (from IR fluxes) $M_{\odot} \mathrm{yr}^{-1}$.

Some authors, as Condon et al. (2002), classified Mkn 1087 as Active Galactic Nucleus (AGN) but our data do not support it. The spectrum of the central zone of Mkn 1087 (see Fig. 4) is similar to the ones of typical starburst galaxies. The FWHM of the $\mathrm{H} \beta$ and [O III] $\lambda 5007$ emission lines are $223 \mathrm{~km} \mathrm{~s}^{-1}$ and $228 \mathrm{~km} \mathrm{~s}^{-1}$ (corrected for instrumental broadening), respectively. The emission line widths in nuclear $\mathrm{H}$ II regions and starburst galaxies have values up to $\sim 300 \mathrm{~km} \mathrm{~s}^{-1}$, whereas the typical $F W H M$ median value range for AGNs is between 350 and $550 \mathrm{~km} \mathrm{~s}^{-1}$ (Véron et al. 1997). Furthermore, we can make use of the Dopita et al. (2000) diagnostic diagrams based on emission line ratios to classify the excitation mechanism of the emission-line systems. In Fig. 11 these relations are plotted together with our observational data, being evident that the knots studied here are consistent with the loci of typical HII regions and not with AGNs. AGNs are clustered around $\log ([\mathrm{O} \mathrm{III}] / \mathrm{H} \beta) \sim 1, \log ([\mathrm{N} \mathrm{II}] / \mathrm{H} \alpha) \sim 0.1$ and $\log ([\mathrm{S} \mathrm{II}] / \mathrm{H} \alpha) \sim$ -0.2 (Veilleux \& Osterbrock 1987).

However, Mkn 1087 could be classified as a Luminous Compact Blue Galaxy (LCBG). LCBGs are $\sim L^{\star}\left(L^{\star}=1.0 \times\right.$ $\left.10^{10} L_{\odot}\right)$, blue, high surface brightness, high metallicity, vigorous starbursting galaxies with an underlying older stellar population (Guzmán et al. 1998). Specifically, a LCBG has $(B-V)<0.6$, a surface brightness $\mu_{B}<21 \mathrm{mag} \mathrm{arcsec}^{-2}$ and $M_{B}<-18.5$. Mkn 1087 satisfies all these properties (for a radius of $5 \operatorname{arcsec}$, it has $(B-V)=0.11$, a $\mu_{B} \sim 21 \mathrm{mag} \mathrm{arcsec}^{-2}$ and $\left.M_{B}=-22.3\right)$. LCBGs are quite common at intermediate 
redshifts, but by $z \sim 0$ their number density has decreased by a factor of ten and seem to have, on average, an order-ofmagnitude smaller masses that typical galaxies today with similar luminosities (Guzmán et al. 2003). The observed properties in LCBGs at low $z$ are similar to the high $z$ Lyman-break galaxies (Erb et al. 2003). Consequently, LCBGs are especially interesting for studies of galaxy evolution and formation because they could be the equivalent of the high $z$ Lyman-break galaxies in the local universe and they have evolved more than other galaxy class.

\subsection{WR population}

We have not detected neither the blue Wolf-Rayet bump nor the nebular He II $\lambda 4686$ emission line in the spectrum of the central zone of Mkn 1087. However, we have used the data obtained by Vaceli et al. (1997) to derive the WR population in the galaxy, assuming that all the flux came from the He II $\lambda 4686$ emission line. These authors estimated that $F(\mathrm{H} \beta)=7.81 \times 10^{-14} \mathrm{erg} \mathrm{s}^{-1} \mathrm{~cm}^{-2}$ and $F(\mathrm{He}$ II $\lambda 4686)=$ $8.36 \times 10^{-15} \mathrm{erg} \mathrm{s}^{-1} \mathrm{~cm}^{-2}$. If we consider that all the contribution of the He II $\lambda 4686$ emission line comes from WNL we find around $7200 \mathrm{WNL}$ stars assuming that $L(\mathrm{WNL} 4686)=$ $1.7 \times 10^{36} \mathrm{erg} \mathrm{s}^{-1}$ for a WNL star (Vacca \& Conti 1992). This value is somehow intriguing, because Vacca \& Conti (1992) reported an upper limit of 1600 to the total WNL presented in Mkn 1087, a factor 4.5 smaller than our estimate. Are really there Wolf-Rayet stars? The slit used by Vaceli et al. (1997) was $2.4^{\prime \prime} \times 4.8^{\prime \prime}$, whereas the one used by Vacca \& Conti (1992) was $1.5^{\prime \prime}$ wide, but they do not indicate its long. We used a $1^{\prime \prime} \times$ $5.4^{\prime \prime}$ aperture in our WHT observations. Perhaps, the WR stars are concentrated in a very small area and Vaceli et al. (1997) were the only ones observing at the correct place.

To derive the $\mathrm{WR} /(\mathrm{WR}+\mathrm{O})$ ratio, we have to correct the contribution of the WR stars to the ionizing flux to obtain the total number of $\mathrm{O}$ stars, assuming that $L(\mathrm{H} \beta)=$ $4.76 \times 10^{36} \mathrm{erg} \mathrm{s}^{-1}$ for a O7V star (Vacca \& Conti 1992) and $\eta \equiv \mathrm{O} 7 \mathrm{~V} / \mathrm{O}=0.25$ (Schaerer \& Vacca 1998). We derived around $47500 \mathrm{O}$ stars in Mkn 1087, that implies a $\mathrm{WR} /(\mathrm{WR}+\mathrm{O})$ ratio of 0.13 . We have also used the calibration of the $\mathrm{WR} /(\mathrm{WR}+\mathrm{O})$ ratio using the flux of the blue WR bump given by Schaerer \& Vacca (1998) in their study about evolutionary synthesis models for $\mathrm{O}$ and WR populations in young starburst (their Eq. (17)). Assuming that all the blue WR bump flux comes from the He II $\lambda 4686$ emission line and using the He II $\lambda 4686$ flux from Vaceli et al. (1997), we estimate a $\mathrm{WR} /(\mathrm{WR}+\mathrm{O})$ ratio of 0.12 , a value in excellent agreement with our previous determination. However, these values represent an upper limit to the real one because Vaceli et al. (1997) did not specify the stellar or nebular origin of the He II $\lambda 4686$ emission line.

The derived age (see Sect. 4.1 and Table 3) for the youngest bursts of star formation in Mkn 1087: knots C (the central zone) and \#7, between 4 and $6 \mathrm{Myr}$, suggest that WR stars should be present in so young massive starbursts. Aperture effects seem to be playing an important role in the no-detection of WR features.

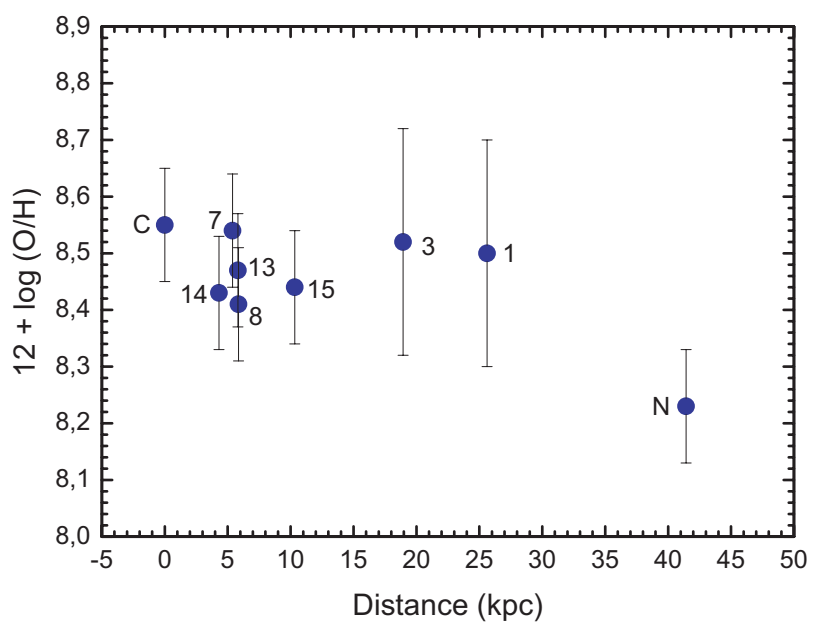

Fig. 12. Relation between metallicity and distance from the nucleus of Mkn 1087 for all the observed knots. We have considered the oxygen abundances derived using the calibration by Pilyugin (2001).

\subsection{The chemical composition of the objects.}

We have analyzed the radial dependence of the derived metallicity of the knots in Mkn 1087, which is shown in Fig. 12. We have used the oxygen abundance obtained from the empirical calibration of Pilyugin (2001) (see Table 6), although this method gives a high uncertain oxygen abundance for knots \#1 and \#3 (we have assumed an error of \pm 0.20 dex for both, and \pm 0.10 dex for the rest of the objects, see Sect. 3.2.2).

We can distinguish two zones in Fig. 12: the first one is the galaxy disk itself, that extends to around $10 \mathrm{kpc}$ from the center of Mkn 1087. We find 5 different objects in this area (the inner knots), two of them (C and \#7) observed both with INT and WHT (we use the average value of both independent determinations). The object \#15 lies just at the border of the disk. The second zone is the external area, where the north companion galaxy and knots \#1 and \#3 are located.

We have explained above that knot \#7 seems to be an intense star-formation zone off-center of Mkn 1087. It is resolved into numerous individual knots in the HST image presented by Malkan et al. (1998). Figure 12 suggests that the oxygen abundances of the center of the galaxy and \#7 are very similar, despite the distance of $6 \mathrm{kpc}$ between them. Keel (1988) suggested that \#7 is the consequence of a merger, but the position-velocity diagrams for PA $357^{\circ}$ and $358^{\circ}$ do not show any dynamical evidence of this. Furthermore, the individual knots that form \#7 in the HST image do not show a clear central concentration, supporting the idea that it is an interaction-triggered star-forming region and not the core of a stripped companion (Walker et al. 1996). The oxygen abundance of the different knots along the disk of Mkn 1087 is rather similar, but the central zone shows a slightly higher value $[12+\log (\mathrm{O} / \mathrm{H})=8.55$, whereas the external zones show a values around 8.45]. On the other hand, the $\mathrm{N}^{+} / \mathrm{O}^{+}$ratio is about $0.2-0.3$ dex higher in the nucleus. These results suggest the possible presence of a rather weak abundance gradient along the disk of Mkn 1087 (about $-0.013 \mathrm{dex} \mathrm{kpc}^{-1}$ ), although nothing definitive can be said taking into account the 
uncertainties. Typical values of oxygen gradients in spiral galaxies are between $-0.009 \mathrm{dex} \mathrm{kpc}^{-1}$ and $-0.231 \mathrm{dex} \mathrm{kpc}-1$, with an average gradient of $-0.06 \mathrm{dex} \mathrm{kpc}^{-1}$ (Zaritski et al. 1994).

The external knots \#1 and \#3 are located at 26 and $19 \mathrm{kpc}$ (respectively) from the center of Mkn 1087 and seem to show oxygen abundances similar to the ones obtained in the disk of the galaxy $[12+\log (\mathrm{O} / \mathrm{H}) \sim 8.50]$. Taken at face value, this gives further evidence that knots \#1 and \#3 are tidal dwarf galaxies. However, the high uncertainties in their metallicities preclude any definitive conclusion from simple abundance considerations.

From Fig. 12, it is clear that the north companion object has a significant lower $\mathrm{O} / \mathrm{H}$ ratio than the disk of $\mathrm{Mkn}$ 1087. The chemical difference is also evident in the $\mathrm{N}^{+} / \mathrm{O}^{+}$ratio. $\mathrm{N}^{+} / \mathrm{O}^{+}$ is about -1.4 in the companion and between -0.8 and -1.1 in the rest of the knots. The value of -1.4 is of the order of that typical for dwarf galaxies (Garnett et al. 1997). This is a further evidence that the north companion is an external pre-existing galaxy.

\subsection{The luminosity-metallicity relation}

Richer \& McCall (1995) obtained the following relation between absolute $B$ magnitude and oxygen abundance for dwarf irregular galaxies (for $M_{B} \geq-18$, the most common definition for a dwarf galaxy):

$12+\log \frac{\mathrm{O}}{\mathrm{H}}=5.67-0.167 \times M_{B}$

that we have used for the objects in Mkn 1087. In Fig. 13 we show this relation (with an extrapolation to higher luminosities). We have assumed that Mkn 1087 has the metallicity of its center, but obviously its position is under the Richer \& McCall (1995) relation because it is not a dwarf galaxy.

In Fig. 13 we can observe that the position of the north companion is consistent with the metallicity-luminosity relation for dwarf galaxies (in fact, it is the only object that is consistent with it). Therefore, the north companion should be interpreted as an independent nearby dwarf galaxy. The presence of the tidal dwarf \#1 and its associated bridge, which is almost aligned with the companion galaxy, suggests that the north companion is in interaction with Mkn 1087.

Duc \& Mirabel (1998) found that dwarf galaxies with metallicities higher than the ones expected from the metallicity-luminosity relation (i.e., dwarf galaxies located at the upper-right zone in Fig. 13) are TDGs. Knots \#1 and \#3 are located in this zone of the diagram and away from the Richer $\&$ McCall (1995) relation for dwarf irregular galaxies. This behavior is consistent with their possible TDG nature, in the sense that they have a much larger metallicity than it is expected for normal galaxies of the same luminosity. This evidence reinforces our hypothesis that both objects are tidal dwarf galaxies formed from material stripped by Mkn 1087, perhaps due to the interaction with the dwarf north companion object (in the case of \#1 seems likely) and maybe also with the neighbour galaxy KPG 103a.

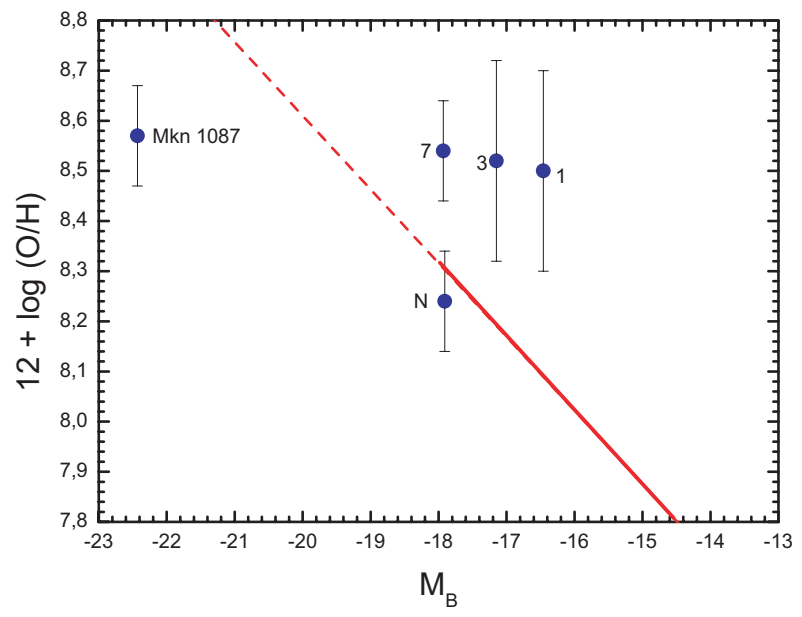

Fig. 13. Luminosity-metallicity relation for the observed detached bursts in Mkn 1087 and knot \#7. The solid line is the relation for dwarf irregulars found by Richer \& McCall (1995), while the dashed line is an extrapolation of it. We have considered the oxygen abundances derived using the calibration by Pilyugin (2001).

Finally, the position of knots \#7 in the metallicityluminosity relation does not support the claim by Keel (1988) that this object is a merged external galaxy. If this is true, the position of the object should be more consistent with the relation.

\subsection{Mkn 1087: A probable group in interaction}

Sometimes, interaction features are found in group of galaxies, especially in Hickson Compact Groups (see, for example, Iglesias-Páramo \& Vílchez 2001; HCG 92: the Stephan's Quintet, Yun et al. 1997; Sulentic et al. 2001; HCG 54, Verdes-Montenegro et al. 2002; NGC 6845 galaxy group, Scott et al. 2003; HCG 31, López-Sánchez et al. 2004). We consider that Mkn 1087, KPG 103a and the north companion object are, in fact, a group of galaxies. We might also include the tidal object \#3 in this group.

We have studied the selection criteria given by Hickson (1982) and Hickson et al. (1992) to define a compact group of galaxies in the particular case of Mkn 1087. Our group satisfied the criteria of the number of members (at least, three galaxies that have similar radial velocities), isolation (there is not other galaxies with $\Delta \mathrm{mag}<3$ in a radii lower than 3 times the assumed size of the group) and compactness criterion (the mean surface brightness of the group, $\mu_{\mathrm{G}}$, defined as the distribution of the flux of all the members over the size of the group, is around 23.5 and, therefore, lower than 26). However, it fails in the relative magnitude criterion because the north companion galaxy differs in more than 3 mag from the absolute magnitude of the brightest galaxies. Consequently, Mkn 1087 and its surroundings can not be strictly classified as a compact group of galaxies attending to Hickson's criteria, which also are defined by the limitations of Hickson's survey. What it is clear to us is that they actually form a group of galaxies with, at least, three members (four, if we also consider object \#3).

The knowledge of the dynamics and galaxy orientations in the group offers some clues to understand what kinds of interactions can lead to the observed structures. Mkn 1087 is a spiral 
galaxy with an inclination of $\sim 37^{\circ}$ with respect to the visual. Its west side is nearer to us and the north side is the approaching one (see Sect. 3.2.3). All the tails seem to be nearly radial to the spiral disk, but the streamers pointing toward objects \#11 and \#12 might be roughly polar, while the tail to knot \#1 could be close to the disk plane. It is intriguing that intense burst \#7 is also aligned in this direction.

Knot \#15, located at the beginning of the long tail to object \#1, shows blue colors, $(U-B) \sim-0.6$, indicating star formation activity. The images (see Figs. 1 and 3 ) reveal small knots along this tail, that decrease in number in the direction to \#1. They seem to be tiny star-forming regions too, because very weak $\mathrm{H} \alpha$ emission is detected in this zone of the WHT spectrum. Knot \#1 appears to be an irregular object composed by diffuse little knots spanning about $4^{\prime \prime}$. We do not think it is a disrupted pre-existing object instead of a tidally stripped remnant because its metallicity is very similar to the spiral disk of Mkn 1087. Furthermore, if knot \#1 were a disrupted low-mass object, its gravitational potential would be strong enough to modify considerably the spiral pattern of Mkn 1087 (Walker et al. 1996). In "minor" mergers, the main galaxy develops a strong two-armed spiral pattern, whereas the nucleus of the low mass external satellite will finally be a small bulgelike entity. Examples of minor mergers are Arp 31 (van Moorsel 1988), NGC 7252 (the Atoms-for-Peace galaxy, Schweizer 1982; Hibbard et al. 1994) or NGC 1097 (Higdon $\&$ Wallin 2003). All these systems show that the inner part of the main galaxy is very disturbed with ripples, loops and tails. None of these features are observed in our case: the HST image of Mkn 1087 reveals a clearly and undisturbed spiral pattern, something that is also observed in the position-velocity diagrams (see Figs. 5 and 6), and knot \#1 is not a bulgelike object. Moreover, Mihos \& Hernquist (1994) proposed that these kind of mergers can lead to a significant inflow of disk gas and fuel a strong central starburst in the main galaxy, being predominantly concentrated in the inner kiloparsec of the disk rather than throughout the disk or bulge. This is not observed in Mkn 1087; the star-formation activity is found all over the disk.

The new data presented through our study give some clues to understand the evolution of the group. The number and intensity of tails and bridges that are observed indicate that interaction processes have been important in the recent past of the system. The gravitational disturbances seem to be the triggering mechanism of the star-formation bursts that nowadays are observed in the knots of Mkn 1087. We propose the following scenario to explain the morphological features and chemical abundances present in the group:

1. Three independent galaxies form the group: Mkn 1087, KPG 103a and the north companion galaxy, this last one is less massive than the others. Mkn 1087 is a spiral galaxy nearly side-on. KPG 103a is probably another spiral galaxy.

2. It is possible that Mkn 1087 and KPG 103a suffered a nearby encounter that formed the tidal features observed towards knots \#11 and \#12 from material stripped from Mkn 1087. We think that it also originated the tidal tail towards knot \#3, that then formed a tidal dwarf galaxy with an important old population (although some star-formation activity also occurred because it shows weak emission features). KPG 103a also developed some type of tidal features that now are difficult to detect; they are barely observed at the east of KPG 103a, although it also shows a diffuse plume towards the west. There are blue knots (possibly, star forming bursts) in the center of the galaxy, suggesting that perhaps KPG 103a is still suffering the effect of tidal perturbations.

3. Some time later, the north companion object probably interacted with the north-east area of Mkn 1087. This encounter formed the tidal tails towards knot \#2 and the intense bridge of material towards knot \#1, perhaps triggering the starburst in both galaxies. This close encounter had to be intense and relatively recent, because the structures that originated them are clearly detected, and were made from material stripped from the main body of Mkn 1087. Some star formation activity is also found in the tail to knot \#1. The very weak plume observed at the south of the north companion galaxy, that points towards knot \#1, reinforces this interacting scenario.

Both interacting processes were probably not timely separated, i.e., perhaps the interaction between Mkn 1087 and the north galaxy began before the interaction with KPG 103a ended. $N$-body simulations (i.e. Mihos \& Hernquist 1996; Dubinski 1998) show that the elliptical galaxies found in most clusters and groups of galaxies have formed through the merger of several galaxies, although galactic cannibalism of smaller galaxies only accounts for a small fraction of the accreted mass. Hence, the probable future evolution of the system will be the merging of all galaxies and, maybe, an elliptical galaxy will be formed. It would be desirable to perform detailed studies of further LCBG in the Local Universe (as Mkn 1087). This could be essential to understanding the origin and evolution of this kind of galaxies in the earlier Universe. Perhaps, galaxy merging and interactions played a more important role at large $z$.

Finally, it should be very interesting to obtain $\mathrm{HI}$ radio map of the system to analyze the distribution of the neutral gas presents in Mkn 1087 and its surroundings (in a similar way to the studies by Hibbard \& van Gorkom 1996; or Hibbard et al. 2001, for example). This study will give a better understanding of the past and future evolution of the group galaxies, as well as it could allow to confirm definitely whether the tidal features observed in optical imagery are originated in the interactions between the different members of this group.

\subsection{The non-catalogued cluster of galaxies}

The cluster of galaxies found at the upper right corner of the image in Fig. 1 is not catalogued in the NASA/IPAC Extragalactic Database (NED). In Table 8 we show the $B$-magnitude and $(B-R)$ color of some of the background galaxies (labeled with "bg") found in the deep $R$-image and observed in the $B$-image compared with the galaxies belonging to the cluster (labeled with "cl"). We performed a simple analysis of the surface brightness profiles of some of these background galaxies to confirm that they are not star-like objects. Despite 
Table 8. $B$-magnitude (not corrected by reddening) and $(B-R)$ color of some of the background galaxies (bg) found in the area surrounding Mkn 1087. The objects belonging to the non-catalogued cluster (cl) show redder colors.

\begin{tabular}{ccc}
\hline \hline Object & $m_{B}$ & $(B-R)$ \\
\hline bg1 & $18.49 \pm 0.08$ & $0.61 \pm 0.12$ \\
bg2 & $19.21 \pm 0.08$ & $0.36 \pm 0.12$ \\
bg3 & $19.43 \pm 0.08$ & $0.29 \pm 0.12$ \\
bg4 & $19.67 \pm 0.08$ & $0.70 \pm 0.12$ \\
bg5 & $20.6 \pm 0.1$ & $0.4 \pm 0.2$ \\
bg6 & $21.5 \pm 0.2$ & $0.4 \pm 0.3$ \\
bg7 & $21.6 \pm 0.2$ & $0.1 \pm 0.3$ \\
bg8 & $21.8 \pm 0.2$ & $0.6 \pm 0.3$ \\
\hline cl1 & $20.8 \pm 0.1$ & $1.1 \pm 0.2$ \\
cl2 & $21.8 \pm 0.2$ & $1.0 \pm 0.3$ \\
cl3 & $21.9 \pm 0.2$ & $1.8 \pm 0.3$ \\
cl4 & $22.0 \pm 0.2$ & $2.3 \pm 0.3$ \\
c15 & $22.5 \pm 0.2$ & $2.7 \pm 0.3$ \\
cl6 & $23.2 \pm 0.3$ & $2.8 \pm 0.5$ \\
\hline
\end{tabular}

the uncertainties due to the faintness of the galaxies with $m_{B}>$ 20 , the objects of this non-catalogued cluster show redder colors that the rest of the background galaxies.

It is possible to determine the redshift to the cluster using photometric redshift codes, for example, using the Fernández-Soto et al. (1999) or HyperZ (Bolzonella et al. 2000) codes, that find the redshift of each object using a fitting procedure that compares the observed magnitudes with the expected ones from standard spectral energy distributions (SED). However, to apply these methods to our cluster, it should be necessary to get the magnitude in, at least, another broad-band filter. Nevertheless, using the code that is been developed by Juncosa \& Gutiérrez (some previous results can be found in Juncosa et al. 2004), we estimate a redshift between 0.2 and 0.4 for it. The study of redshift clusters gives important clues about both galaxy and cluster formation and evolution.

\section{Conclusions}

We have used deep optical and near-infrared imagery and deep optical intermediate-resolution spectroscopy to analyze the morphology, colors, physical conditions, kinematics and chemical abundances of the galaxy Mkn 1087 and its surrounding objects. We have obtained the oxygen abundances for several star-forming bursts using empirical calibrations. We have obtained consistent age estimations for the knots observed in Mkn 1087 using different observational indicators and theoretical models.

We have derived a Keplerian mass of $\sim 5.6 \times 10^{10} M_{\odot}$ for the main body of Mkn 1087. We have detected a new companion object at the north, which has an oxygen abundance and $\mathrm{N} / \mathrm{O}$ ratio lower that the rest of the knots, a Keplerian mass of $\sim 2.2 \times 10^{8} M_{\odot}$, an old underlying stellar population, and it is detached of the rotation pattern of the main galaxy. All these evidences suggest that the north companion object is an external dwarf galaxy. We suggest that some of the objects (\#1, $\# 2, \# 3, \# 11$ and \#12) are TDG candidates.

Mkn 1087 is not powered by an AGN but it could be classified as a LCBG because of its color, absolute magnitude and surface brightness. Wolf-Rayet features are not detected in the brightest bursts, perhaps due to aperture effects. Luminous knot \#7 hosts a strong starburst that should not be the consequence of a merger, more likely it is a complex of star-forming regions located in a spiral arm of Mkn 1087.

In conclusion, the complex geometry of the filamentary structure of Mkn 1087 and all the photometric, chemical and kinematical results can be explained assuming that it is in interaction with two external galaxies:

1. The relatively bright KPG $103 \mathrm{a}$, that could explain the bridges, the non-stellar objects located between both galaxies (\#11 and \#12), and the tidal dwarf galaxy \#3.

2. And the new dwarf north companion, that could originate the tidal features towards knot \#2, the bridge between Mkn 1087 and the knot \#1, and produce the star formation triggering in the knots found in them.

We consider that Mkn 1087 and its surroundings should be classified as a group of galaxies in interaction.

Finally, we have also serendipitously detected a noncataloged cluster of galaxies with central position around $\alpha=04^{\mathrm{h}} 49^{\min } 35^{\mathrm{s}}, \delta=+03^{\circ} 21^{\prime} 04^{\prime \prime}$ and probably at a redshift between 0.2 and 0.4 .

Acknowledgements. We are very grateful to the referee, Bill Keel, for his very useful comments. We would like to acknowledge José A. Caballero for his macro for NIR reduction and Víctor Sánchez-Béjar for his help with CST observations. We thank Vahram H. Chavushyan for helpful comments on the characteristics of AGNs. We are very grateful to Jorge García-Rojas for his help in the derivation of the helium abundances and to Robert Juncosa for his valuable comments about photometric redshifts. M.R. acknowledges support from Mexican CONACYT project J37680-E. This research has made use of the NASA/IPAC Extragalactic Database (NED) which is operated by the Jet Propulsion Laboratory, California Institute of Technology, under contract with the National Aeronautics and Space Administration.

\section{References}

Allende Prieto, C., Lambert, D. L., \& Asplund, M. 2001, ApJ, 556, 63 Benjamin, R. A., Skillman, E. D., \& Smits, D. P. 2002, ApJ, 569, 288 Bettoni, D., Galletta, G., \& García-Burillo, S. 2003, A\&A, 405, 5

Bolzonella, M., Miralles, J.-M., \& Pelló, R. 2000, A\&A, 363, 476, HyperZ

Brandt, J. C. 1960, ApJ, 131, 553

Brocklehurst, M. 1971, MNRAS, 153, 471

Calzetti, D., Armus, L., Bohlin, R. C., et al. 2000, ApJ, 533, 682

Campos-Aguilar, A., Moles, M., \& Masegosa, J. 1993, AJ, 106, 1784

Castellanos, M. 2000, Ph.D. Thesis: A comprehensive Study of High Metallicity Giant Extragalactic H II Regions, Univ. Autónoma de Madrid

Condon, J. J., Cotton, W. D., \& Broderick, J. J. 2002, AJ, 124, 675

Conti, P. S. 1991, ApJ, 377, 115

Denicoló, G., Terlevich, R., \& Terlevich, E. 2002, MNRAS, 330, 69 
Dopita, M. A., Kewley, L. J., Heisler, C. A., \& Sutherland, R. S. 2000, ApJ, 542, 224

Dubinski, J. 1998, ApJ, 502, 141

Duc, P.-A., \& Mirabel, I. F. 1998, A\&A, 338, 813

Erb, D. K., Shapley, A. E., Steidel, C. C., et al. 2003, ApJ, 591, 101

Esteban, C., López-Sánchez, A. R., \& Rodríguez, M. 2003, in A Massive Star Odyssey: From Main Sequence to Supernova, ed. K. van der Hucht, A. Herrero, \& C. Esteban (San Francisco: ASP), IAU Symp., 212, 712

Fernández-Soto, A., Lanzetta, K., \& Yahil, A. 1999, ApJ, 513, 34

Garnett, D. R. 1992, AJ, 103, 1330

Garnett, D. R., Shields, G. A., Skillman, E. D., Sagan, S. P., \& Dufour, R. J. 1997, ApJ, 489, 63

Gordon, D., \& Gottesman, S. T. 1981, AJ, 86, 161

Guzmán, R., Jadren, A., Koo, D. C., et al. 1998, ApJ, 495, L13

Guzmán, R., Östlin, G., Kunth, D., et al. 2003, ApJ, 586, L45

Hibbard, J., Guhathakurta, P., van Gorkom, J. H., \& Schweizer, F. 1994, AJ, 107, 67

Hibbard, J. E., \& van Gorkom, J. H. 1996, AJ, 111, 655

Hibbard, J. E., van der Hulst, J. M., Barnes, J. E., \& Rich, R. M. 2001, AJ, 122, 2969

Hickson, P. 1982, ApJ, 255, 382

Hickson, P., Mendes de Oliveira, C., Huchra, J. P., \& Palumbo, G. G. 1992, ApJ, 399, 353

Higdon, J. L., \& Wallin, J. F. 2003, ApJ, 585, 281

Howarth, I. D., \& Murray, J. 1990, SERC Starlink User Note, No. 50

Hunt, L. K., Mannucci, F., Testi, L., et al. 1998, AJ, 115, 2594

Hunter, D. A., \& Gallagher, J. S. 1985, AJ, 90, 1457

Izotov, Y. I., \& Thuan, T. X. 1999, ApJ, 511, 639

Iglesias-Páramo, J., \& Vílchez, J. M. 2001, ApJ, 550, 211

Juncosa, R., Gutiérrez, C. M., \& Fernández-Soto, A. 2004, in Proc. of the II International Workshop on Science with GTC, Rev. Mex. Astron. Astrofis (SC), in press

Kaler, J. B., \& Lutz, J. H. 1985, PASP, 97, 700

Kauffmann, G., Nusser, A., \& Steinmetz, M. 1997, MNRAS, 286, 795

Keel, W. C. 1988, A\&A, 202, 41

Kennicutt, R. C. 1998, ApJ, 498, 541

Kunth, D., \& Joubert, M. 1985, A\&A, 142, 411

Landolt, A. U. 1992, AJ, 104, 340

Leitherer, C., \& Heckman, T. M. 1995, ApJS, 96, 9

Leitherer, C., Schaerer, D., Goldader, J. D., et al. 1999, ApJS, 123, 3, STARBURST99

López-Sánchez, A. R., Rodríguez, M., \& Esteban, C. 2004, ApJS, accepted

Maeder, A., \& Meynet, G. 1994, ARA\&A, 32, 227

Malkan, M. A., Gorjian, V., \& Tam, R. 1998, ApJS, 117, 25

Marzke, R. O., Huchra, J. P., \& Geller, M. J. 1996, AJ, 112, 1803

Massey, P., Strobel, K., Barnes, J. V., \& Anderson, E. 1988, ApJ, 328, 315

Mazzarrella, J. M., \& Boronson, T. A. 1993, ApJS, 85, 27

Méndez, D. I., \& Esteban, C. 2000, A\&A, 359, 493, ME00

Mihos, J. C., \& Hernquist, L. 1994, ApJ, 425, L13

Mihos, J. C., \& Hernquist, L. 1996, ApJ, 464, 641
Noeske, K. G., Papaderos, P., Cairós, L. M., \& Fricke, K. J. 2003 , A\&A, 410, 481

Olofsson, K. 1995, A\&AS, 111, 57

Okazaki, T., \& Taniguchi, Y. 2000, AJ, 543, 149

Pagel, B. E. J., Edmunds, M. G., \& Smith, G. 1980, MNRAS, 193, 219

Peimbert, M., \& Costero, R. 1969, Bol. Obs. Tonantzintla y Tacubaya, 5,3

Pilyugin, L. S. 2000, A\&A, 362, 325

Pilyugin, L. S. 2001, A\&A, 369, 594

Richer, M. G., \& McCall, M. L. 1995, ApJ, 445, 642

Rieke, G. H., \& Lebofsky, M. J. 1985, ApJ, 288, 618

Sanders, D. B., Soifer, B. T., Elias, J. H., et al. 1988, ApJ, 325, 74

Schaerer, D., \& Vacca, W. C. 1998, ApJ, 497, 618

Schaerer, D., Contini, T., \& Pindao, M. 1999, A\&AS, 136, 35

Schlegel, D. J., Finkbeiner, D. P., \& Davis, M. 1998, ApJ, 500, 525

Schweizer, F. 1982, ApJ, 252, 455

Scott, G., Koribalski, B., \& Jones, K. 2003, MNRAS, 342, 939

Shaw, R. A., \& Dufour, R. J. 1995, PASP, 107, 896

Smith, L. F., Shara, M. M., \& Moffat, A. F. J. 1996, MNRAS, 281, 163

Stasińska, G. 1990, A\&AS, 83, 501

Stasińska, G., Schaerer, D., \& Leitherer, C. 2001, A\&A, 370, 1

Sulentic, J. W., Rosado, M., Dultzin-Hacyan, D., et al. 2001, AJ, 122, 2993

Rubin, V. C., Hunter, D. A., \& Ford, W. K., Jr. 1990, ApJ, 365, 86

Taylor, C., Thomas, d., Brinks, E., \& Skillman, E. D. 1996, ApJS, 107, 143

Telles, E., \& Terlevich, R. 1995, MNRAS, 275, 1

Thuan, T. X. 1991, in Massive stars in starbursts, ed. C. Leitherer, N. R. Walborn, T. M. Heckman, \& C. A. Norman (Cambridge: Cambridge University Press), 183

Thuan, T. X., Izotov, Y. I., \& Lipovetski, V. A. 1995, ApJ, 445, 108

Vacca, W. D., \& Conti, P. S. 1992, ApJ, 401, 543

Vaceli, M. S., Viegas, S. M., Gruenwald, R., \& De Souza, R. E. 1997, AJ, 114, 1345

van Moorsel, G. A. 1988, A\&A, 202, 59

Verdes-Montenegro, L., del Olmo, A., Iglesias-Páramo, J. I., et al. 2002, A\&A, 396, 815

Véron, P., Gonçalves, A. C., \& Véron-Cetty, M.-P. 1997, A\&A, 319, 52

Veilleux, S., \& Osterbrock, D. E. 1987, ApJS, 63, 295

Walker, I. R., Mihos, J. C., \& Hernquist, L. 1996, ApJ, 460, 121

Weilbacher, P. M., Duc, P.-A., \& Fritze-v. Alvensleben, U. 2003, A\&A, 397, 545

Whitford, A. E. 1958, AJ, 63, 201

Wilcots, E. M., Lehman, C., \& Miller, B. 1996, AJ, 111, 1575

Yun, M. S., Verdes-Montenegro, L., del Olmo, A., \& Perea, J. 1997, ApJ, 475, L21

Zaritski, D., Kennicutt, R. C., Jr., \& Huchra, J. P. 1994, ApJ, 420, 87

Zwicky, F. 1971, Catalogue of Selected Compact Galaxies and Post-Eruptive Galaxies, Guemligen 